\title{
MECHANICAL RESPONSE OF NOTCHED MARBLE BEAMS UNDER BENDING VERSUS ACOUSTIC EMISSIONS AND ELECTRIC ACTIVITY
}

\author{
Stavros K. Kourkoulis, Ermioni D. Pasiou, Ioanna Dakanali \\ National Technical University of Athens, Department of Mechanics, Laboratory for Testing and Materials, Athens, Greece \\ e-mail: stakkour@central.ntua.gr \\ Ilias Stavrakas, Dimos Triantis \\ Technological Educational Institute of Athens, Department of Electronics, Laboratory of Electrical Characterization of \\ Materials and Electronic Devices, Athens, Greece
}

\begin{abstract}
An experimental protocol, including the combined application of both innovative and traditional sensing techniques, is described aiming to explore the mechanical response of marble and also to check the possibilities of detecting precursor phenomena designating upcoming catastrophic fracture. The protocol consisted of three-point bending tests with notched prismatic beams made of Dionysos marble, the material extensively used for restoration of the Acropolis of Athens monuments. The sensing system improvised included techniques relying on completely different physical foundations, which permit simultaneous detection and recording of the Pressure Stimulated Currents, Acoustic Emissions, three dimensional displacement fields and Notch Mouth Opening Displacements. Analysis of the results revealed interesting features of the mechanical response of Dionysos marble and indicated, also, that classical Continuum Fracture Mechanics fails to describe accurately the response of marble, at least in the presence of notches. In addition, strong correlations between the Pressure Stimulated Currents, the rate of acoustic hits and the rate of change of the opening of the pre-existing notch have been enlightened. Moreover, the onset of catastrophic crack propagation appears following distinguishable changes of the Pressure Stimulated Currents recorded. Therefore (and taking into account the very small size of the respective sensors as well as the simple complementary equipment needed), it is concluded that the specific technique could be considered as a simple and reliable tool for an alternative approach to the in-situ Structural Health Monitoring of classical stone monuments.
\end{abstract}

Keywords: marble, monuments, notch mouth opening displacement, three-point bending, pressure stimulated currents, acoustic emissions, 3D-digital image correlation

\section{Introduction}

The remaining life and the remaining load-carrying capacity of structural elements seriously concern the engineering community, especially in the case of already damaged or cracked elements. The need for a clear answer to this problem is imperative, among others, for scientists working for conservation projects of ancient stone monuments, given that quite often their structural members are cracked and any extension of pre-existing cracks could be fatal to the structural integrity of the whole monument.

Answering the above question is usually attempted within the frame of Fracture Mechanics by applying various criteria which predict both the load leading a pre-existing crack to initiation and, also, the direction towards which the crack is going to propagate. Such criteria, widely used in engineering praxis, are based on the maximum tangential stress (Yoffe, 1951; Erdogan and Sih, 1963), the strain energy density (Sih, 1973), the maximum energy release rate (Wu, 1978), the maximum dilatational strain energy (Theocaris et al., 1982) etc. In the restoration 
praxis of stone monuments, however, the problem is far more complicated due to the anisotropic nature of building materials and the fact that the loading schemes acting on a given element are not easily represented according to standard load-simulation schemes used in the laboratory. Therefore, the classical crack initiation criteria of Fracture Mechanics are not directly applicable, and it is not realistic to expect that closed analytic answers could be obtained. In addition, the direct "transfer" of results from laboratory experimental studies to the structural engineering field is questionable due to the "size effect" (Bažant, 1984; Carpinteri, 1989), i.e., the dependence of material properties on the specimen size. The "size effect" is extremely pronounced in rock and rock-like materials like, for example, those used for the construction of classical monuments of Cultural Heritage in Greece (Sulem and Vardoulakis, 1990; Kourkoulis and Ganniari-Papageorgiou, 2010).

Taking into account the above difficulties, it appears that in-situ continuous Structural Health Monitoring (SHM) of stone monuments is the only preventive tool that could provide on-time warning about upcoming changes, which could be harmful for the structural integrity of such monuments. However, it should be clarified from the very beginning that SHM of monuments of Cultural Heritage is not a trivial task. In order to understand the challenging character of the venture, one could consider the Parthenon Temple in Athens. The structural elements of the most emblematic monument of the "Golden Age of Pericles" (epistyles, column drums, capitals) were made of marble blocks quarried from the Pentelic Mountain. As it can be seen in Fig. 1a, quite a few of these elements are seriously cracked and their load-carrying capacity is questionable (Korres and Bouras, 1983; Zambas, 1994), rendering continuous SHM an imperative demand. The coexistence of authentic and substitute marble (Fig. 1a) together with metallic connectors and cement paste (used to fill the grooves (Fig. 1b) in which the metallic connectors are placed) causes additional difficulties. This coexistence creates internal (hidden) interfaces, along which failure/damage mechanisms are firstly activated, well before any crack extension (or any other damage mode) is observable at the outer surface of the member.

(a)

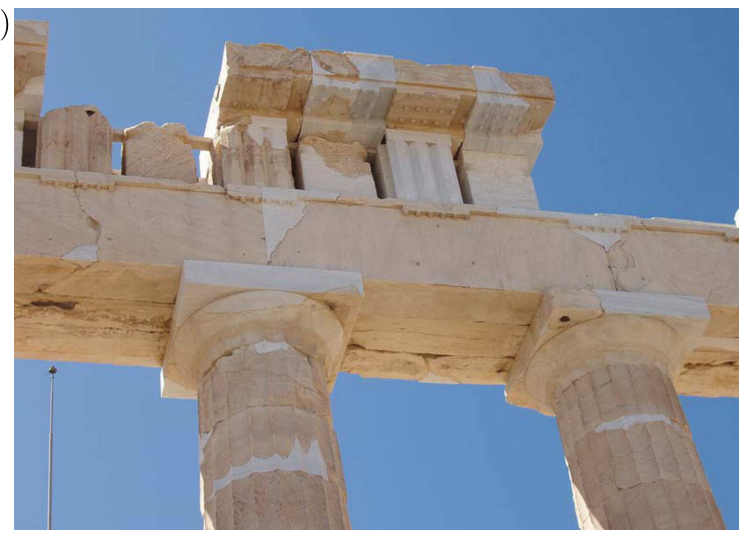

(b)

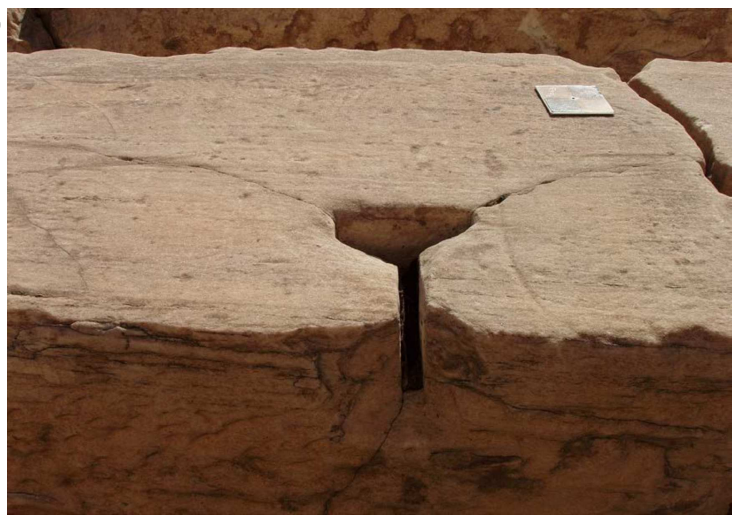

Fig. 1. (a) Typical epistyles of the Parthenon Temple showing the degree of damage and, also, the coexistence of authentic elements (made of Pentelic marble) with patches made of the substitute material (Dionysos marble) joined together by means of invisible metallic elements. (b) The grooves sculptured by ancient Greeks to host the metallic elements used to keep series of epistyles in place

In the frame of the above argumentation, it is easily concluded that questions related to the remaining load-carrying capacity of restored structural members of stone monuments could not be answered unless data from the interior of the element are pumped. Moreover, it is clear that such data can only be pumped using sensors of very small size (to avoid harming the aesthetic harmony of the monument) and also of low cost (considering the number of sensors required to monitor all the cracked structural elements of a given monument, which for the case of the Parthenon Temple are too many). Such sensing systems, widely used today, both in 
laboratory and structural scale, include detection of acoustic events, application of optical fibers, quantification of electric resistance changes etc. Their in-situ application is relatively difficult, and for some of them their application necessitates interventions on the monument members, which are usually not permissible.

In this direction, the present study aims to comparatively assess the efficiency of a relatively novel sensing technique, which is based on the detection of extremely weak electric currents, known as the Pressure Stimulated Currents (PSC) (Triantis et al., 2006, 2012). Among the critical advantages of the PSC technique is the very small size of the respective sensors and their low cost as well as the simple complementary equipment of the set-up, rendering the technique quite attractive, especially for long term monitoring of many structural members.

For the study to be accomplished, notched prismatic marble beams are subjected to Three-Point Bending (3PB). The PSC produced is recorded as a function of time, in parallel with the data provided by the Acoustic Emission (AE) technique, which is considered a well-founded and mature sensing technique, widely used worldwide. In addition, the displacement field developed is recorded using the 3D-Digital Image Correlation (3D-DIC) technique while the Notch Mouth Opening Displacement (NMOD) is also recorded using a clip-gauge extensometer.

The data obtained from this combined "attack" (i.e., with both innovative and traditional experimental techniques) are comparatively studied in the direction of correlating critical aspects of their time evolution. Interesting conclusions are drawn about the mechanical behaviour and failure of Dionysos marble, while the possibility of using the critical Notch Opening Displacement (NOD) as a simple easy-to-apply fracture criterion is discussed. Moreover, strong correlations between the PSC, the AE as well as the data obtained using the 3D-DIC technique (properly assessed against the ones of the traditional clip-gauge extensometer) are highlighted. The time evolution of the above quantities was proven to follow internal damage processes in a satisfactory manner. In addition, indications were provided, according to which the time evolution curves of all the quantities mentioned above exhibited changes (either clear or imperceptible) that could be considered as pre-failure indicators well before macroscopic crack initiation.

\section{The experimental protocol}

\subsection{The material and the specimens}

The specimens of the experimental protocol were cut out from an almost cubic block of marble quarried from Dionysos Mountain in Attica region. Given that the specific variety of marble is usually considered as a transversely isotropic material, every effort was made for all the specimens to be cut along the strong anisotropy direction and the load to be applied normally to the material layers, in the direction of minimizing the scattering of the results. In general, every effort was made to control (if not to eliminate) the factors responsible for the scattering of the experimental results. All specimens were cut out from the same marble block and they were carefully inspected for visible defects. Moreover, they were cut along the same direction with respect to the material layers, and the notches were machined carefully and by a single technician. After completion of the experimental protocol, Chauvenet's criterion was used to exclude from the elaboration of the results all tests for which the deviations were unacceptably increased. As a result, the final scattering of the tests included in the analysis was quite affordable (at least for tests with specimens made of a brittle rock-like material).

The specimens were beam-shaped with a rectangular cross section and overall length equal to $L_{o}=100 \mathrm{~mm}$. Two classes of specimens were tested concerning the dimensions of the cross section: a class with width-to-height ratio equal to $b \times h=20 \times 20 \mathrm{~mm}^{2}$ and the second one with $b \times h=25 \times 25 \mathrm{~mm}^{2}$. The specimens were mechanically notched at their mid-span with the aid of a cutting disc of thickness $\delta_{o}=2.5 \mathrm{~mm}$ and rounded cutting edge. The length of the notch 
was constant and equal to $\alpha_{o}=4 \mathrm{~mm}$ resulting to two notch length-over-specimen height ratios, i.e., $\alpha_{o} / h=0.20$ and $\alpha_{o} / h=0.16$, respectively, in an effort to check also the role of the relative notch length. The geometry and dimensions of the specimens are shown in Fig. 2b.

(a)

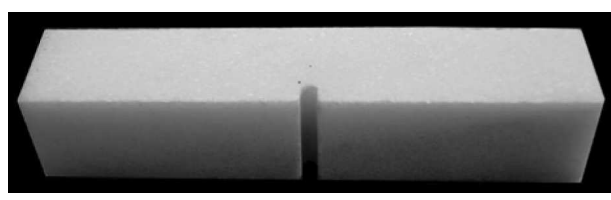

(b)

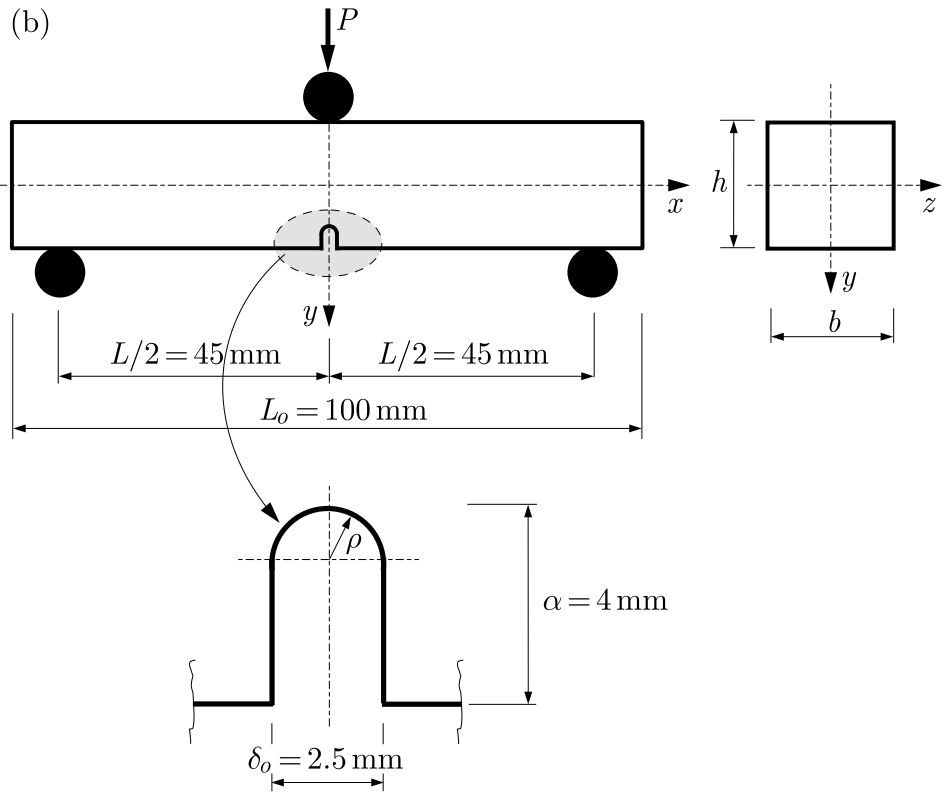

Fig. 2. (a) A typical specimen before testing. (b) The geometry and dimensions of the specimens

Dionysos marble was chosen because it is the material used by the scientific and technical personnel of the restoration project of the Athens Parthenon Temple for constructing copies of missing structural elements and also for preparing patches for partially destroyed members. The project is still in progress and is implemented by the "Acropolis Restoration Service" under the auspices of the "Committee for the Conservation of the Acropolis Monuments", an interdisciplinary committee of the Greek Ministry of Culture, established in 1975. The methodology adopted and the restoration techniques developed in the frame of the project are worldwide recognized for their pioneering nature and the strict commitment to the restoration principles of "The Venice Charter" (1964).

The properties (mechanical and physicochemical) of Dionysos marble are very similar to those of the authentic building stone of the Parthenon Temple, i.e., Pentelic marble. It is an extremely fine-graded white marble consisting of calcite (about 98\%) and very small amounts of muscovite, sericite, quartz and chlorite. It is of almost white colour with a few thin ash-green veins along the marble's schistosity. Silver areas are detected due to the presence of chlorite and muscovite. Its porosity is very low (varying between $0.3 \%$ and $0.7 \%$ ). Its density is equal to about $2730 \mathrm{~kg} / \mathrm{m}^{3}$ and its absorption coefficient by weight varies around $0.11 \%$. The coefficient of thermal expansion is $9 \cdot 10^{-6 \circ} \mathrm{C}$ (between $15^{\circ} \mathrm{C}$ and $100^{\circ} \mathrm{C}$ ). The grain size varies around $0.43 \cdot 10^{-3} \mathrm{~mm}$ and the crystals are polygonic of almost uniform size (between $900 \mu \mathrm{m} \times 650 \mu \mathrm{m}$ and $950 \mu \mathrm{m} \times 874 \mu \mathrm{m})$ (Tassogiannopoulos, 1986).

The mechanical properties of Dionysos marble reported in literature vary within broad limits. This is because they depend, among others, on the exact quarrying point and depth as well as on the orientation of the specimens with respect to the anisotropy directions. The numerical values adopted in this study are based on an exhaustive experimental study by Vardoulakis and Kourkoulis (1997) and are recapitulated in Table 1, for the strong anisotropy direction, which is of importance for the present study. 
Table 1. Mechanical properties of Dionysos marble along the strong anisotropy direction (Vardoulakis and Kourkoulis, 1997)

\begin{tabular}{|l|c|c|c|c|c|c|c|}
\hline Property & \multicolumn{2}{|c|}{ Young's modulus } & $\begin{array}{c}\text { Tensile } \\
\text { strength }\end{array}$ & $\begin{array}{c}\text { Compressive } \\
\text { strength }\end{array}$ & $\begin{array}{c}\text { Nominal tensile } \\
\text { strength }\end{array}$ & $\begin{array}{c}\text { Poisson's } \\
\text { ratio }\end{array}$ \\
\hline \hline Test & Tension & $\begin{array}{c}\text { Comp- } \\
\text { ression }\end{array}$ & $3 \mathrm{~PB}$ & $\begin{array}{c}\text { Tension- } \\
\text {-Brazilian }\end{array}$ & $\begin{array}{c}\text { Uniaxial } \\
\text { compression }\end{array}$ & $3 \mathrm{~PB}$ & $\begin{array}{c}\text { Tension- } \\
\text {-compression }\end{array}$ \\
\hline Unit & $\mathrm{GPa}$ & $\mathrm{GPa}$ & $\mathrm{GPa}$ & $\mathrm{MPa}$ & $\mathrm{MPa}$ & $\mathrm{MPa}$ & - \\
\hline Value & 75 & 84 & 109 & 9.1 & 78.4 & 18.4 & 0.23 \\
\hline
\end{tabular}

\subsection{The sensing techniques used}

\subsubsection{Pressure Stimulated Currents}

It has been long ago observed that mechanical stress is responsible for generation of electric signals in brittle materials like rocks. It was Whitworth (1975), in a pioneering work, who demonstrated such an effect in alkali halides. It is worth mentioning, also, that electric signals are generated when brittle non-piezoelectric materials (the porosity of which does not favour electro-kinetic phenomena) are subjected to an abrupt increase of the level of mechanical stress. Nowadays, the most widely accepted model describing qualitatively the generation of these weak electrical signals is the one introduced by Slifkin (1993). The specific model was further developed by Vallianatos and Tzanis (1999) and is commonly designated as the Moving Charged Dislocations (MCD) model.

According to the MCD model, motion of charged dislocations produces a transverse electric polarization $P$ directly related to the electric current density $J$ and, in turn, to the mechanical strain rate, $d \varepsilon / d t$

$$
J=\frac{\partial P}{\partial t} \propto \frac{d \varepsilon}{d t}
$$

The above transient electrical signals are detected in the form of electric currents, known as Pressure Stimulated Currents (PSC), and the respective experimental technique is known as the PSC technique (Triantis et al., 2006). It was used for the first time by Varotsos (2005) in the direction of describing the polarization or depolarization of electric signals, as a result of pressure variations on solids that contain dipoles due to the existence of defects. According to Eq. (2.1), it is expected for the PSC to be related to the accumulation of deformation and more specifically to be proportional to the mechanical strain rate $(d \varepsilon / d t)$.

The technique has been applied successfully to rock samples (made either of marble (Stavrakas et al., 2003) or amphibolite (Triantis et al., 2007)), submitted to monotonically increasing compressive load until fracture. Later on, it was applied, also successfully, to cement based specimens under compression (Kyriazopoulos et al., 2011; Triantis et al., 2012).

Experimental studies indicate that the PSC emitted during mechanical loading has a deterministic form and can be used as a tool for detecting the upcoming fracture. Moreover, the form of the PSC can be used to distinguish whether plasticity (non-reversible deformation of the specimen) has appeared or whether fracture is impending (Anastasiadis et al., 2007). It is generally observed that the PSC emissions increase intensively when the applied mechanical stress approaches the strength limit of the tested specimen (Triantis et al., 2012). Experiments with specimens made of cementitious materials indicated that mechanical straining due to axial compression induces electric signals, providing qualitatively similar results with those in rocks and rock-like materials (Kyriazopoulos et al., 2011). However, the PSC recorded is significantly stronger than that in rocks, reaching amplitudes at the $\mathrm{nA}$ scale. Moreover, recent experiments with prismatic specimens made either of marble or mortar and subjected to $3 \mathrm{~PB}$ revealed that 
proper evaluation of the electric current emission recordings may provide useful information regarding the proximity of the applied mechanical load to the ultimate stress level of the specimens (Stergiopoulos et al., 2015).

In the laboratory, the PSCs are detected by using extremely sensitive electrometers. The sensors consist of pairs of gold-plated electrodes attached on the specimen's surface by means of a conductive paint. In the present study, the measuring system consisted of an ultra-sensitive programmable electrometer (Keithley, $6517 \mathrm{~A}$ ) resolving currents from $0.1 \mathrm{fA}$ to $20 \mathrm{~mA}$ in 11 ranges. The data of the electrometer were stored in a computer using GPIB interface. The electrodes were installed in the notch and on the upper side of the specimens (Fig. 3a). The overall arrangement of the equipment of the PSC system used is shown in Fig. 4.

(a)

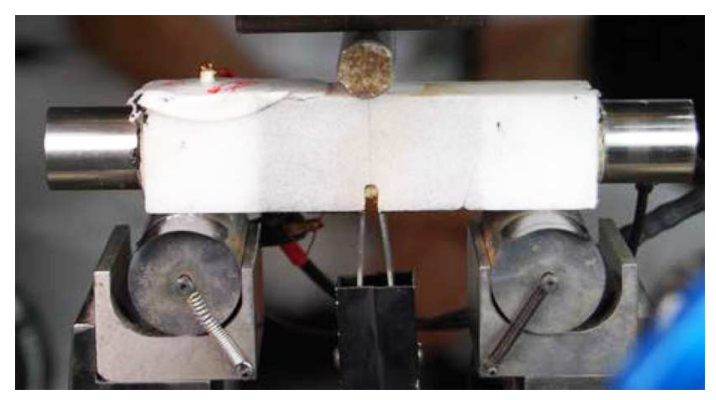

(b)

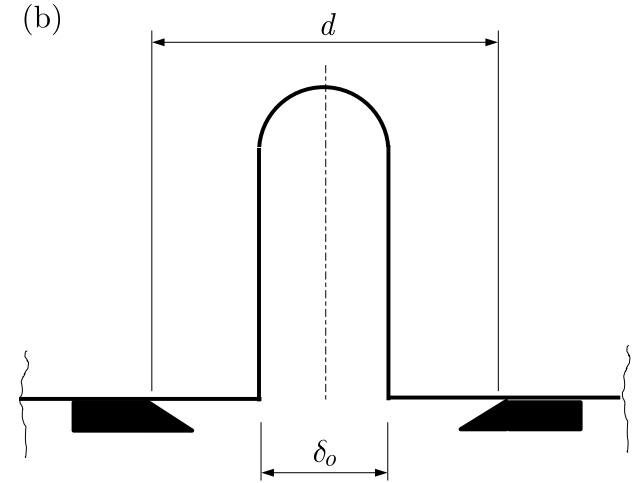

(c)

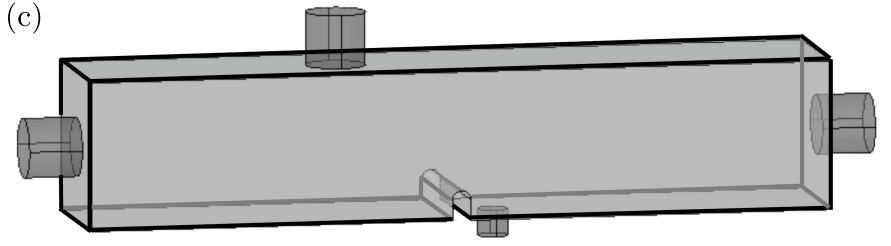

Fig. 3. (a) A specimen placed on the metallic rollers just before testing. (b) A detailed sketch of the knife-edges used to keep the clip-gauge in place. (c) A schematic representation of the position of the Acoustic Emission sensors

\subsubsection{Acoustic Emissions}

The Acoustic Emission (AE) technique detects acoustic events taking place within a material during mechanical loading, since deformation and failure of materials are accompanied by a sudden release of strain energy. The energy released generates elastic stress waves which travel within the material towards the boundaries of the specimen, where they are detected by proper transducers (usually piezoelectric sensors converting elastic waves to electrical signals). The main advantage of the $\mathrm{AE}$ technique is that it can monitor failure processes during the whole loading scheme of either a specimen or a whole structure by just attaching a number of sensors on it. Recalling that acoustic emissions depend mainly on irreversible deformations, it is concluded that this technique is suitable for structural health monitoring. Another advantage of the AE technique is related to its ability to determine the spatial coordinates of the acoustic source employing usually the travel-time-difference method (Sachse et al., 1991).

The underlying principle of the technique is dated back to 1933, when the process of shock occurrence in a wood specimen under flexural loading was studied by Kishinoue with the aid of a phonograph pick-up and a steel needle. His original article (in Japanese) was translated into English by Ono in 1990 (Kishinoue, 1990). The first studies concerning the acoustic emissions in geomaterials were carried out by Obert (1941). Till today, the AE technique has been applied to various materials, i.e., rocks, concrete, mortars, metals etc. under various loading 
(a)

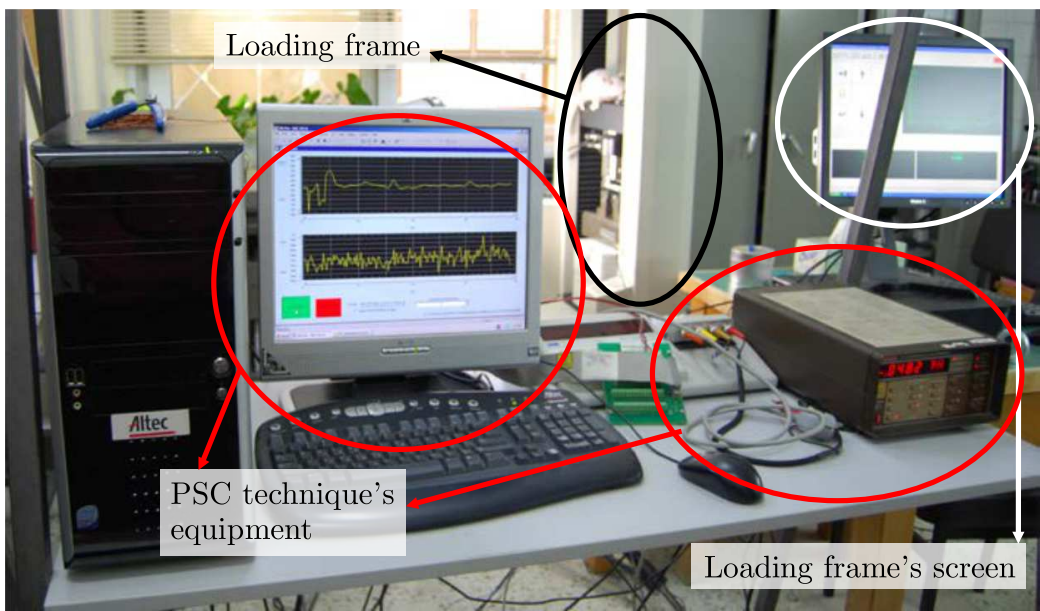

(b)

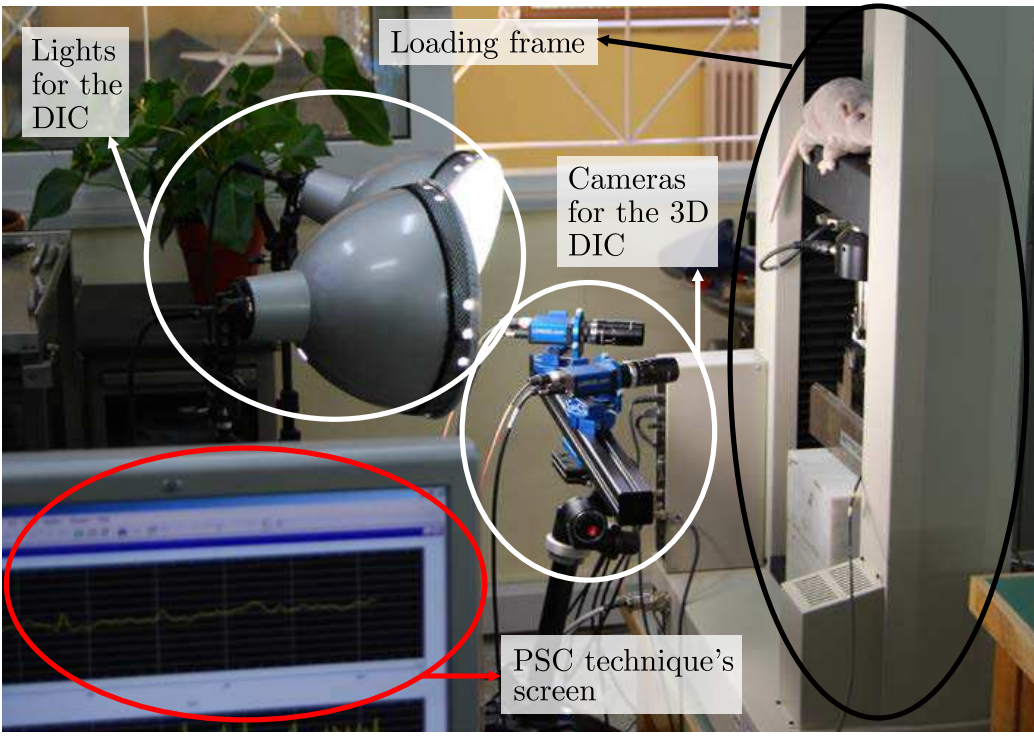

Fig. 4. An overview of the experimental set-up. (a) The equipment for recording the PSC. (b) The arrangement for the application of the DIC technique

modes. Maji and Shah (1988), for example, determined the fracture process zone in pre-cracked concrete specimens under tension by means of the source location of acoustic emissions. Labuz et al. (2001) quantified the intrinsic process zone in cementitious specimens of various size subjected to diametral compression (Brazilian-disc test) and 3PB. The dimensions of the fracture process zone based on the location of the acoustic events were determined also for rock specimens (Muralidhara et al., 2010). Another very interesting application of the AE technique is the estimation of the critical values of the SIF and the J-integral, dated back to 1984 (Blanchette et al., 1984). Later on, Hashida (1993) applied the same approach for rock-like materials. Recently, the $\mathrm{AE}$ technique has been used for the classification of the failure modes, also, in rock-like materials. For example, bending tests were carried out by Aggelis et al. (2013) in marble specimens, in an effort to correlate the frequency and the waveform shape of the AE with the fracture mode.

Quite often the data recorded by the AE sensors are analyzed using the $b$-value (Rao and Prasanna Lakshmi, 2005), correlating events of high amplitude and lower frequency with events of low amplitude and higher frequency. Nowadays, an "Improved $b$-value" concept ( $I b$-value), proposed by Shiotani et al. (2001), is employed. It is based on statistical parameters (such as the mean and standard deviation of the AE amplitude), which vary during the test. The 
time variation of the $I b$-values is considered as providing valuable indications concerning the proximity of the system to its "critical stage" (impending failure). The $I b$-value is defined as

$$
I b=\frac{\log _{10} N\left(\mu-\alpha_{1} \sigma\right)-\log _{10} N\left(\mu+\alpha_{2} \sigma\right)}{\left(\alpha_{1}+\alpha_{2}\right) \sigma}
$$

where $\sigma$ is the standard deviation and $\mu$ is the mean value of the AE amplitude distribution. Moreover, $\alpha_{1}$ is related to the smaller amplitude while $\alpha_{2}$ is related to the fracture level. The numerical values of $\alpha_{1}, \alpha_{2}$ vary within broad limits $\left(0.5<\alpha_{1}, \alpha_{2}<5.0\right)$. Attributing to them values within this range does not significantly affect the $I b$-value (Shiotani et al., 1994). In this context, it is here assumed that $\alpha_{1}=\alpha_{2}=1.0$.

Besides providing valuable information concerning the intensity of the internal damage process and its spatiotemporal evolution, it is generally accepted, nowadays, that the data recorded by the $\mathrm{AE}$ technique offer interesting information concerning the nature of the source of acoustic signals (Ohno and Ohtsu, 2010; Ohtsu, 2010; Aggelis, 2011; Aggelis et al., 2013). More specifically, the relationship between the RA parameter (Rise Time/Amplitude) and the Average Frequencies (AF) (counts/duration) permits classification of the cracks formed during loading to Mode I and/or Mode II. According to this approach, acoustic emission signals of high AF and low RA values are due to "tensile" (mode I) cracks. On the other hand, acoustic emission signals of low AF and high RA values are due to other crack modes (mode II crack or mixed-mode cracks) or even due to shear phenomena (friction).

In the present study, four acoustic emission sensors were properly attached on the free transverse sections of the specimens with the aid of silicone. Three of the sensors were of the R15 $\alpha$ type while the fourth one was a pico-sensor. The positions of the sensors are shown schematically in the sketch in Fig. 3c.

\subsubsection{Digital Image Correlation}

The third innovative technique used is the Digital Image Correlation (DIC). It is based on the combined use of two high definition cameras. Its theoretical background is dated 30 years ago (Sutton et al., 1986), and it has been since then continuously developed further (Sutton et al., 2000). For its application, the surfaces monitored are covered by a dense grid of paint dots the displacements of which during loading are used to calculate the full-field 3D displacement-field. DIC has been already used for the determination of Crack Tip Opening Displacement and Stress Intensity Factors (SIFs) of brittle materials (Brynk et al., 2012).

In this study, a novel 3D-DIC system (LIMESS Messtechnik \& Software GmbH, Germany) with two cameras (of resolution $1624 \times 1234$ pixel) was used. The 3D-DIC active field was a rectangle of dimensions $44 \times 27 \mathrm{~mm}^{2}$. The size of the pattern dots was equal to about $0.08 \mathrm{~mm}$. The frequency was set to 1 photo every 3 seconds. The 3D-DIC system used in the present study is shown in Fig. 4.

\subsection{The experimental set-up and the experimental protocol}

The experiments were implemented using an MTS-INSIGHT loading frame of capacity 10 $\mathrm{kN}$. In parallel to the above mentioned techniques, a traditional clip-gauge extensometer (INSTRON 2670-120) with a $5 \mathrm{~mm}$ gauge length and $2 \mathrm{~mm}$ range was properly mounted to the notch mouth (Figs. 3a,b) to measure the Notch Mouth Opening Displacement (NMOD). It was calibrated using a micrometer calibrator (High Mag). The TDS-530 data-logger was used for data acquisition and storage. An overview of the whole experimental set-up is shown in Fig. 4.

The specimens were placed on two metallic cylinders of an INSTRON 3PB fixture (Fig. 3a). The diameter of the supporting cylinders was $25 \mathrm{~mm}$ and their distance was adjusted (and kept constant for all tests) to $L=90 \mathrm{~mm}$, resulting to a span-over-height ratio approaching 4 . The 
specific value is considered as the lower limit for which the Bernoulli-Euler technical bending theory gives acceptable results, permitting one, in the first approximation, to ignore the influence of shear forces.

The tests were quasi-static realized under displacement-control mode at a rate equal to $0.01 \mathrm{~mm} / \mathrm{min}$. The load was applied monotonically until fracture of the specimens. It was linearly distributed along the width of the specimens with the aid of the third steel cylinder of diameter $10 \mathrm{~mm}$ (Fig. 3a).

\section{Fracture characteristics of Dionysos marble under 3PB}

All specimens tested were fractured in the same way: A crack was initiated at the tip of the notch and propagated towards the load application point, almost parallel to the direction of the applied load (Fig. 5a). In some specimens, the crack path deviated slightly (Fig. 5b) indicating some kind of asymmetries of the whole configuration. Considering that every attention was paid to the experimental set-up to be symmetric, it can be concluded that those deviations were due to inevitable local inclinations of the material layers of marble with respect to the specimen's longitudinal axis. The fracture surfaces were almost planar (although slightly wavy) and normal to the longitudinal axis of the specimens, as it can be seen in Figs. 5c and 5d. In general, the fracture plane is very sensitive to local material imperfections (typical for most marble

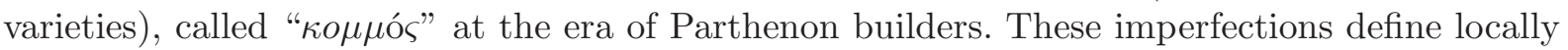
weak material planes. Obviously, such specimens were not taken into account while elaborating the results of the experiments.

(a)

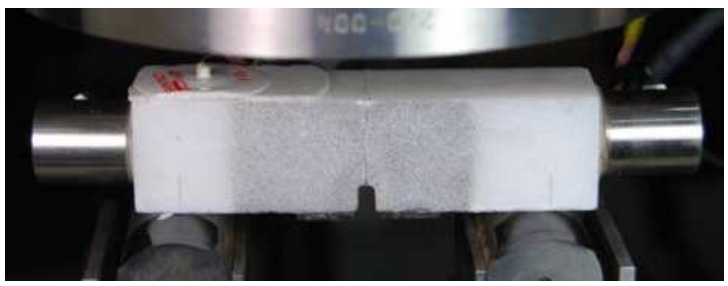

(b)

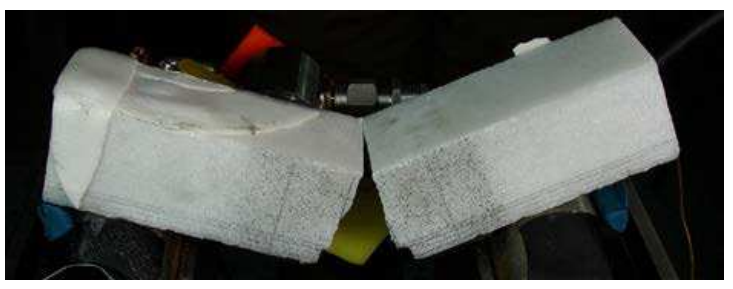

(c)

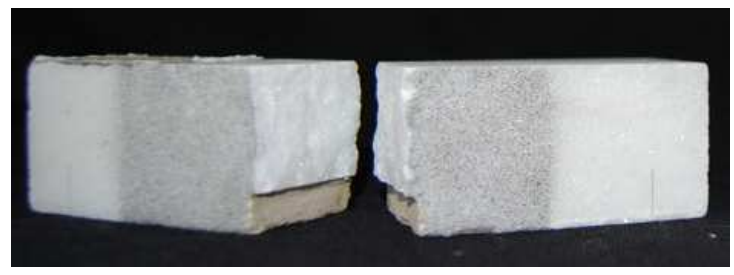

(d)

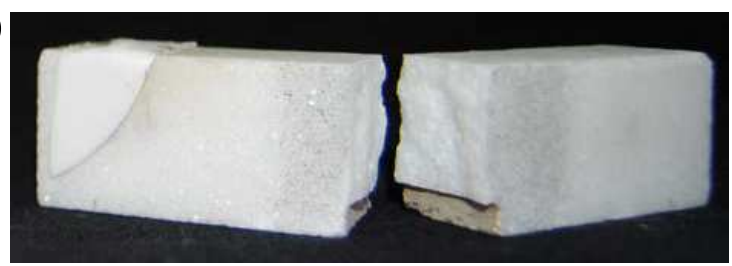

Fig. 5. Typical fractured specimens with fracture planes either parallel to the loading axis (a) or slightly inclined with respect to this axis (b). The wavy shape of typical fracture surfaces (c), (d)

Typical raw data for the variation of the load imposed versus the respective deflection of the central cross section (as provided by the translation of the frame traverse) are plotted in Fig. 6, for some characteristic specimens belonging to both specimen classes. Ignoring inevitable bedding errors, all curves are more or less similar to each other, approaching linearity in a quite satisfactory manner for the whole loading procedure. The fracture load for both specimen classes (around $600 \mathrm{~N}$ for the class with $b \times h=20 \times 20 \mathrm{~mm}^{2}$ and around $1100 \mathrm{~N}$ for the class with $b \times h=$ $25 \times 25 \mathrm{~mm}^{2}$ ) exhibited relatively low scattering in spite of the increased number of unpredictable parameters that could increase scattering for materials like marble (inhomogeneity, anisotropy, internal defects etc.). The maximum deflection of the central cross section was determined equal to about $0.10 \mathrm{~mm}$ for the specimens of the first class and equal to about $0.16 \mathrm{~mm}$ for those of the second class, again with a relatively low standard deviation. 


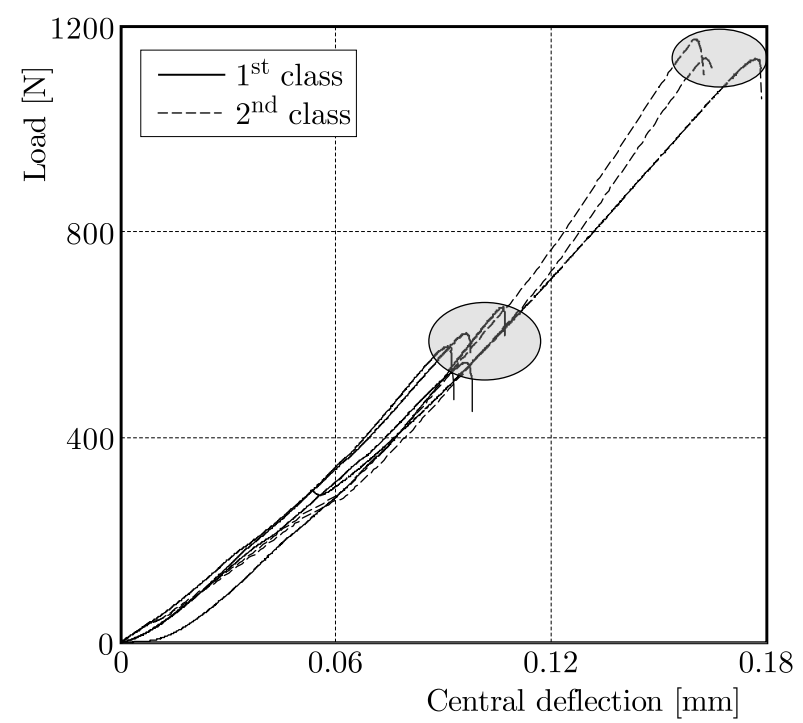

Fig. 6. Raw data of some characteristic experiments of both classes of specimens: variation of the load induced against the displacement of the loading frame traverse

The variation of the Notch Mouth Opening Displacement (NMOD), as it was obtained by the clip-gauge, normalized over the initial distance between the knife edges of the clip-gauge (Fig. 3b), is plotted in Fig. 7 with respect to the maximum "nominal" axial stress developed, also, for some characteristic specimens. The "nominal" stress was calculated by the familiar formula of the classic Bernoulli-Euler technical bending theory (taking into account the effective cross sectional area)

$$
\sigma_{n o m}=\frac{3 P L_{o}}{2 b\left(h-\alpha_{o}\right)^{2}}
$$

where $P$ is the force applied. It is seen that after a more or less linear portion these graphs become non-linear, contrary to what is perhaps expected for a material of an increased brittleness like Dionysos marble. It is here clarified that the data of Fig. 7 for the NMOD are plotted up to the maximum stress value. Clearly, the clip-gauge continues recording even after the peak stress is reached, since the fracture of the specimen and its fragmentation into two pieces is macroscopically observed with some delay after the stress attains its maximum level. This phenomenon is the origin of quite a few problems, related to the accurate determination of the critical value of NMOD, in case the latter is considered as a critical quantity (criterion) describing the fracture. Due to its importance, the specific point will be discussed further in Section 5. The average value of the dimensionless critical NMOD for Dionysos marble was found equal to about $7.5 \cdot 10^{-4}$.

The average value of the "nominal" axial fracture stress, in the presence of a notch, was 14.04 MPa with a relatively low (considering the brittleness of the material) standard deviation. The differences between the specimens of the two classes are negligible, indicating that it is the presence of the notch itself rather than its length that dictates the final outcome. The respective value for the "nominal" axial fracture stress of intact Dionysos marble beams under 3PB, as it was determined by Vardoulakis et al. (1997), is equal to 18.4 MPa. Both values (i.e., for notched and intact specimens) are considerably higher than the respective one determined from direct tension tests, reported again by Vardoulakis et al. (1998), which for the strong anisotropy direction was equal to $9.1 \mathrm{MPa}$ (Table 1). This conclusion supports further the indications concerning the inappropriateness of the $3 \mathrm{~PB}$ test as a substitute of the direct tension test.

What is to be noted, also, from Fig. 7, is that the stress-NMOD curves deviate from linearity from relatively early load levels, contrary to the respective load-deflection curves. This could 


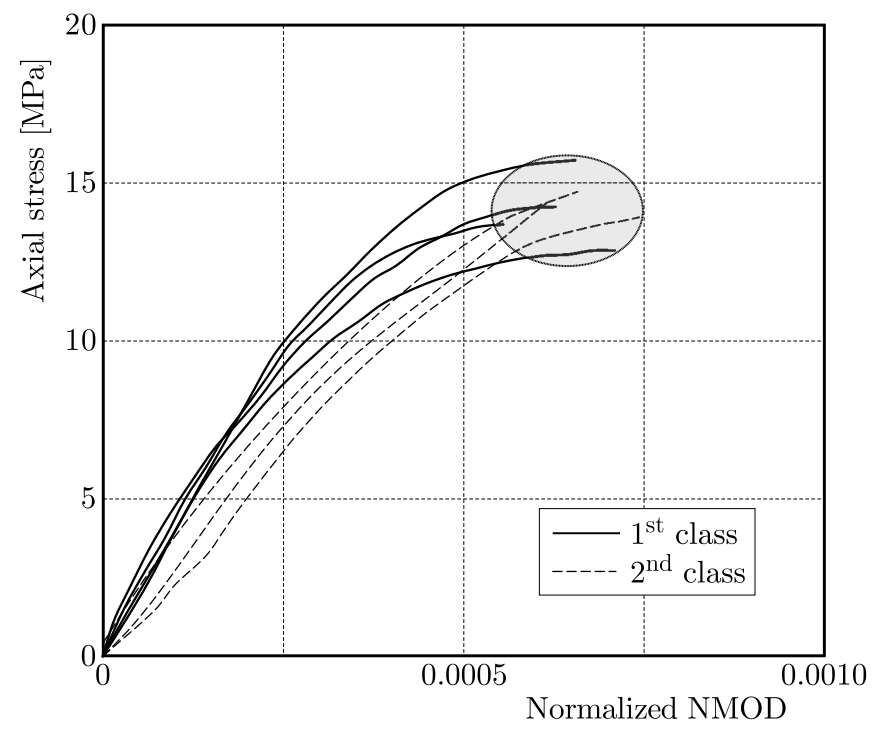

Fig. 7. The variation of the longitudinal "nominal" axial stress against the Notch Mouth Opening Displacement as obtained by the clip-gauge. The values of NMOD are normalized over the initial distance $d$ between the knife edges of the clip-gauge

be explained by the fact that the NMOD strongly depends on the stress field at the immediate vicinity of the notch crown, where the so called "process" zone is formed and the material locally ceases obeying the linearity assumption. On the other hand, the overall deflection depends on the global stress field all over the specimen. Taking into account that the maximum size of the process zone for Dionysos marble is of the order of only a few millimetres (Kourkoulis et al., 1999), it is reasonable to assume that it cannot drastically influence the overall response of the specimen.

In addition, it should be emphasized that the slope of the experimentally obtained stressNMOD curves is much more abrupt compared to that obtained analytically using the familiar formula for the stress-NMOD relation for a beam with a crack, as it is provided by classical Fracture Mechanics for a beam with length-to-height ratio equal to four (Tada et al., 1973)

$$
\begin{aligned}
& \delta=\frac{4 \sigma_{n o m} \alpha}{E^{\prime}} V_{1}\left(\frac{\alpha_{o}}{h}\right) \\
& V_{1}\left(\frac{\alpha_{o}}{h}\right)=0.76-2.28\left(\frac{\alpha_{o}}{h}\right)+3.87\left(\frac{\alpha_{o}}{h}\right)^{2}-2.04\left(\frac{\alpha_{o}}{h}\right)^{3}+\frac{0.66}{\left(1-\frac{\alpha_{o}}{h}\right)^{2}}
\end{aligned}
$$

In Eqs. (3.2) $E^{\prime}$ is the equivalent Young's modulus of the beam material (depending on whether plane stress or plane strain conditions prevail). The difference exceeds $100 \%$, and it is quite impossible to be addressed to any type of experimental error or to the fact that the length-to-height ratio of the specimens tested was not exactly equal to four for the second class of specimens (it was equal to 3.6). In fact, Eq. (3.2) is only valid for "mathematical" cracks (zero distance between the crack lips) and only approximately describes the stress-NMOD relation in the case of notched specimens. Therefore, it should be used with caution, since it ignores the role of the radius of curvature $\rho$ (see Fig. 2b) of the crown of the notch, which under specific circumstances could be critical (Markides and Kourkoulis, 2016). Moreover, as it was pointed out by Vardoulakis et al. (1998) and Kourkoulis et al. (1999), 3PB of beams made of Dionysos marble should be described in the frame of gradient elasticity, taking into account the internal microstructure of the specific material. The latter is the only approach permitting description of the size-effect, which is quite pronounced for the specific material. 
Before concluding this Section, two additional aspects of the mechanical behaviour of Dionysos marble should be mentioned, which render the theoretical analysis of $3 \mathrm{~PB}$ in the frame of Linear Elastic Fracture Mechanics even more prone to large discrepancies from experimental reality. The first one is the slight non-linearity characterizing the axial stress-axial strain curve of the specific marble type. The second one is its bimodularity, i.e., the fact that its elastic modulus under tension is not identical to that under compression (Exadaktylos et al., 2001).

Interesting conclusions about the deformed shape of the specimens just before fracture can be drawn from Fig. 8 in which the deflections of both the upper and lower edges of a typical specimen are plotted for its central part from either side of the notch. The deflections are obtained from the data of the DIC system. Any rigid body translation and rotation was removed. It is observed from Fig. 8 that the deformed contours of the upper and lower edges are not self similar. This behaviour could be attributed to:

(i) The presence of the notch and

(ii) The decrease of the specimen height due to the relatively short length of the specimen (and the way the load is applied, i.e., to the punch effect).

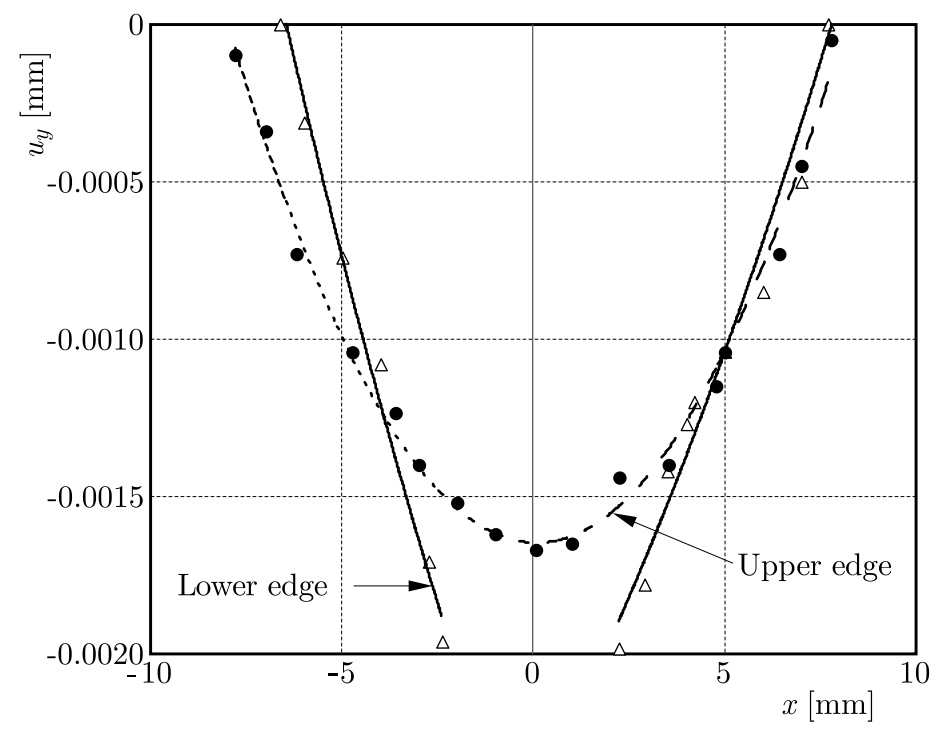

Fig. 8. The vertical (parallel to the load direction) displacement of the upper (filled cycles) and lower (empty triangles) edges of a typical specimen for its central part, as they were obtained with the aid of the 3D-DIC technique (rigid body translations and rotations are removed)

Both these factors are responsible for generation of compressive vertical normal stresses along the height of the central part of the specimens. In Fig. 8, the best fit curves of the experimental data are also shown (continuous lines). For both the upper and lower edges, it is concluded that their deformed shapes are simulated by second order functions of the form

$$
y=c_{1} x^{2}+c_{2} x+c_{3}
$$

with a very satisfactory correlation factor $R^{2}$ ranging from 0.97 to 0.99 . In Eq. (3.3) $c_{1}, c_{2}$ and $c_{3}$ are constants obtained numerically with the aid of commercial software.

As the next step, the experimental data for the NMOD, as obtained from both the DIC technique and the clip-gauge, are discussed. A typical view of the field of axial displacements $u_{x}$ (used for the indirect determination of NMOD) is shown in Fig. 9a, as it was captured by the DIC technique just before the final macroscopic fracture of a typical specimen. The impending cracking path is clearly detectable as the locus of zero $u_{x}$-values. In order to take advantage of the specific DIC data, two small polygons were isolated on both sides of the notch at its 
lowest level and the distance between them was determined as a function of time. Removing the initial distance between the centroids of the two polygons and also removing any rigid body displacements, one obtains the NMOD. The raw data for the time variation of the NMOD as it is obtained from both the DIC technique and the clip-gauge are plotted in Fig. 9b for a typical specimen. Careful inspection of this figure indicates that, although the DIC data are qualitatively similar to the respective ones of the clip-gauge, some quantitative differences exist, mainly for relatively low load levels, where the DIC data appear underestimating the respective NMOD values recorded by the clip-gauge. However, at the ultimate load steps (which are of utmost importance for the determination of the critical NMOD value) the data of the two techniques are very close to each other. In general, according to both techniques, it is only during the very last load steps that the NMOD starts increasing rapidly according to an almost exponential law, and the respective graphs become almost vertical. As a result, the determination of the critical NMOD becomes extremely difficult. Similar conclusions were obtained experimentally by Andrianopoulos et al. (1997) for some Metal Matrix Composites under direct tension, although they adopted a completely different recording technique. Saragas et al. (1996) published additional data supporting the present conclusions and, moreover, they provided some exponential laws describing accurately enough the dependence of NMOD on the stress level.
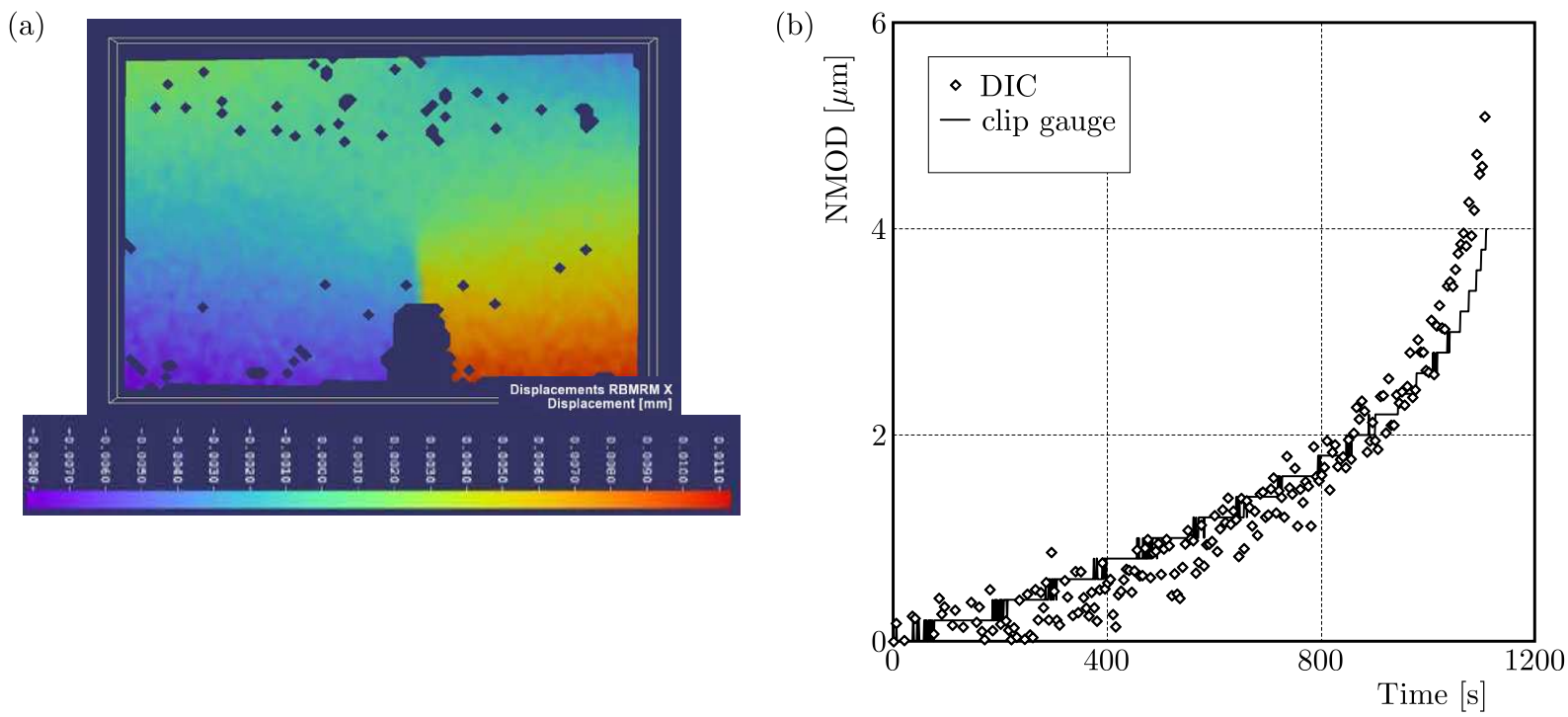

Fig. 9. (a) Axial displacements as recorded by the 3D-DIC technique. The crack path to be followed is visible as the locus with the zero axial displacement. (b) The time variation of the NMOD as it was measured by the clip-gauge (continuous line) versus that recorded by the 3D-DIC system (empty rhomboid symbols)

The deformed notch contour can be deduced from Fig. 10a, in which the horizontal displacements of the points of a locus $s$, closely surrounding the notch are plotted against the variable $s$ (the contour of the specific locus approaches the actual boundary of the notch as close as it is permitted by the resolution of the DIC system). It is interesting to observe that the flanks of the notch remain linear, thus justifying a geometric extrapolation of the NMOD values in order to obtain the Notch Opening Displacement (NOD). The maximum value of this quantity is in fact the geometry-independent parameter that could be potentially used as fracture criterion. Taking advantage of Fig. 10a, it can be concluded that the critical NMOD for Dionysos marble (at least for the block tested in the present protocol) is equal to about $7.5 \mu \mathrm{m}$ (after removing the initial distance between the knife-edges of the clip-gauge). Then, adopting the procedure shown in Fig. 10b (Knauf and Riedel, 1981) with $\varphi=60^{\circ}$, the respective value for the critical NOD (which according to some fracture criteria could be considered as a material constant) is found equal to about $4.4 \mu \mathrm{m}$. 
(a)

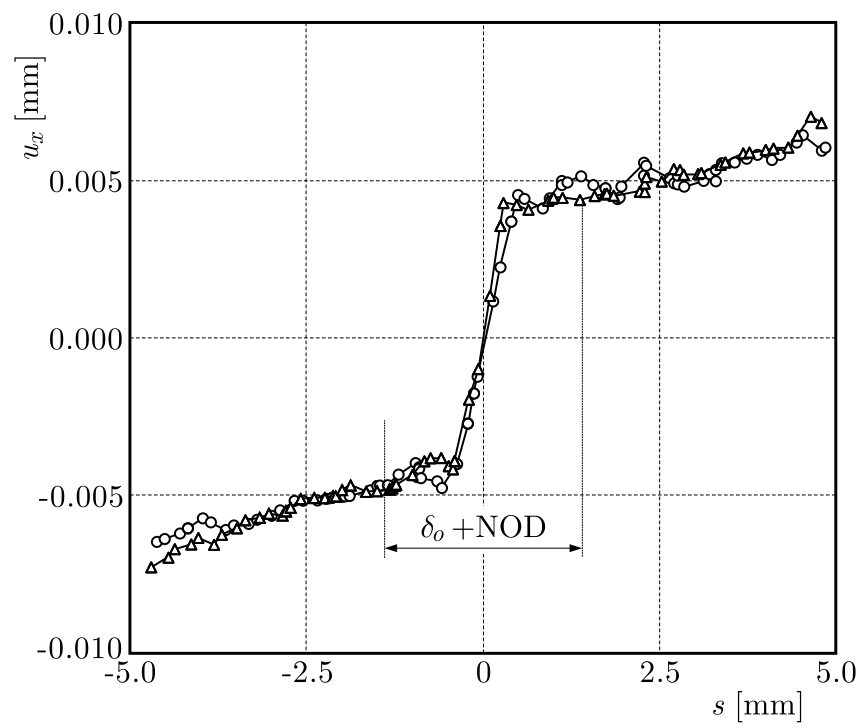

(b)

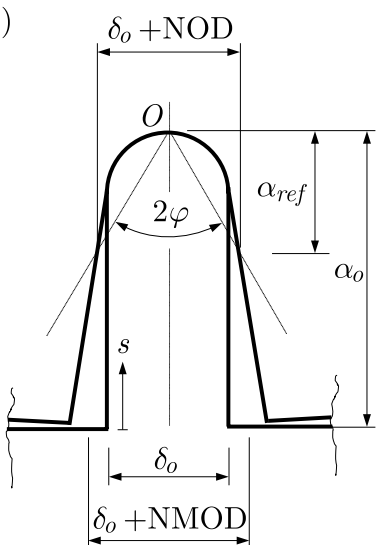

Fig. 10. (a) Axial (horizontal) displacements of a linear locus $s$ closely surrounding the notch (as close as the resolution of the DIC system permits). (b) The procedure adopted to determine the Notch Opening Displacement based on data for the respective NMOD

\section{Acoustic and electric activity during three-point bending}

\subsection{An overview of the whole loading procedure}

The time variation of the PSC, recorded during the whole loading process, is plotted in Fig. 11a, in juxtaposition to the respective variation of the load induced, for a representative specimen with a cross section equal to $b \times h=20 \times 20 \mathrm{~mm}^{2}$. It is seen that during the initial loading steps (i.e., for load levels lower that $50 \%$ of the fracture load) the PSC increases very smoothly, in fact it is almost constant. At a load level equal to about $50 \%$ of the fracture load (point $A$ in Fig. 11a), the magnitude of the PSC starts increasing at a different (much higher) rate while the respective load-time plot exhibits an imperceptible slope change (see the shadowed rectangle in Fig. 11a). According to the literature (Triantis et al., 2006, 2007), the onset of an intense PSC increase designates the onset of micro-cracking within the volume of the specimen and, therefore, it can be concluded that point $A$ corresponds to the onset of intense damage processes. Moreover, at a time instant $t_{1}$, a little before the load attains its ultimate value, it is observed that the value of PSC holds back instantaneously (point $B$ in Fig. 11a). The specific point indicates the generation of a local macro-crack which interrupts instantaneously the local electric paths. From this point on, it is considered that the system (specimen) entered its "critical stage", and final fracture is impending (Stergiopoulos et al., 2015; Triantis et al., 2012).

In Fig. 11b, the aforementioned quantities are plotted for a characteristic experiment of the second class, i.e., with a specimen of the cross section equal to $b \times h=25 \times 25 \mathrm{~mm}^{2}$. Although the PSC-time curve exhibits qualitatively similar behaviour to that of Fig. 11a, there are some interesting differences: While at the very early loading levels the PSC-value is again almost constant, it starts increasing relatively earlier (see point $A$ in Fig. 11b), following an "anomaly" of the respective load-time curve. This "anomaly" is characterized by a slight drop of the load-time curve, which then keeps increasing but with a clearly different slope (see the shadowed rectangle in Fig. 11b). Again, at a time instant $t_{1}$, a little before the load attains its ultimate value, the PSC holds back instantaneously (point $B$ in Fig. 11b), indicating again that the system entered its "critical stage" and fracture is impending. 

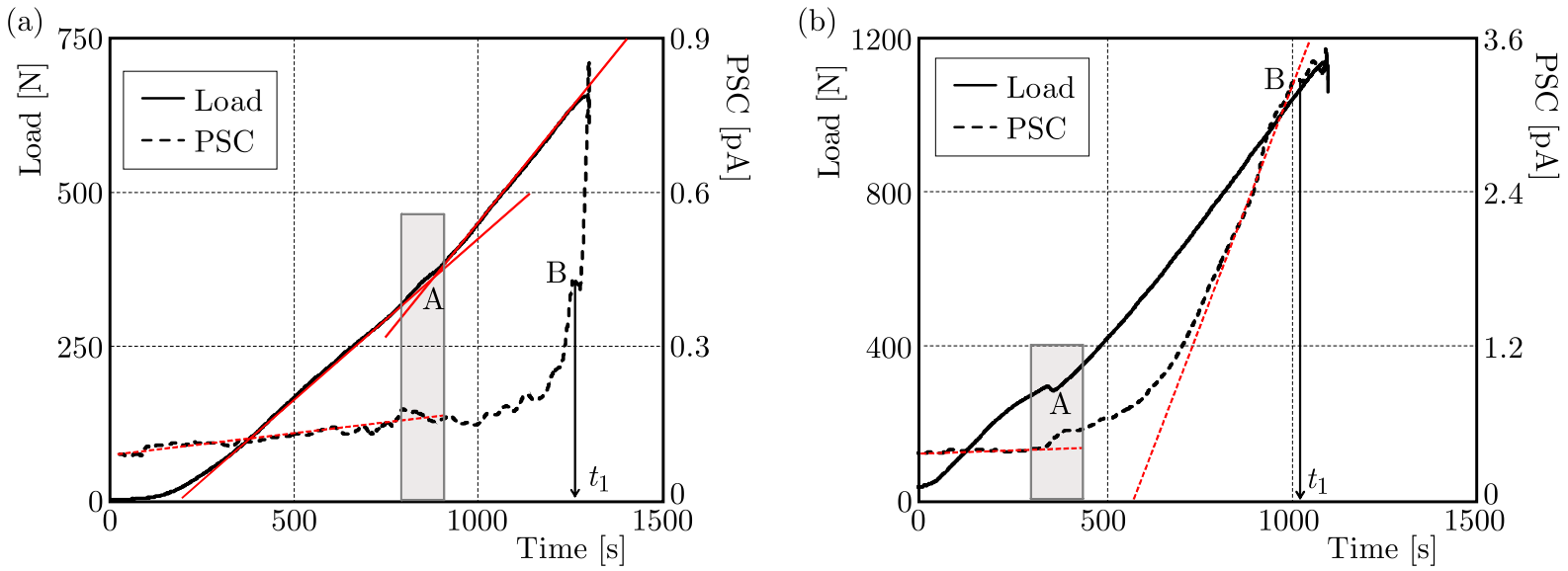

Fig. 11. The time evolution of the load induced in juxtaposition to the respective one of the PSC recorded for a specimen with $b \times h=20 \times 20 \mathrm{~mm}^{2}$ (a) and $b \times h=25 \times 25 \mathrm{~mm}^{2}$ (b)

Considering the overall behaviour of the two specimens just discussed (and besides any differences which are expected due to the inhomogeneous and anisotropic nature of the material studied), it can be concluded that the time-variation of the PSC offers characteristic indications concerning both the onset of intense micro-cracking within the specimen volume and also the entrance of the system (specimen) to its "critical stage". Moreover, it is worth noticing that the above indications are not accompanied by similar indications of the load-time curve, which is more or less a continuous and monotonous line (see also Fig. 6) with imperceptible slope changes which do not offer clear hints of the upcoming catastrophic failure.

(a)

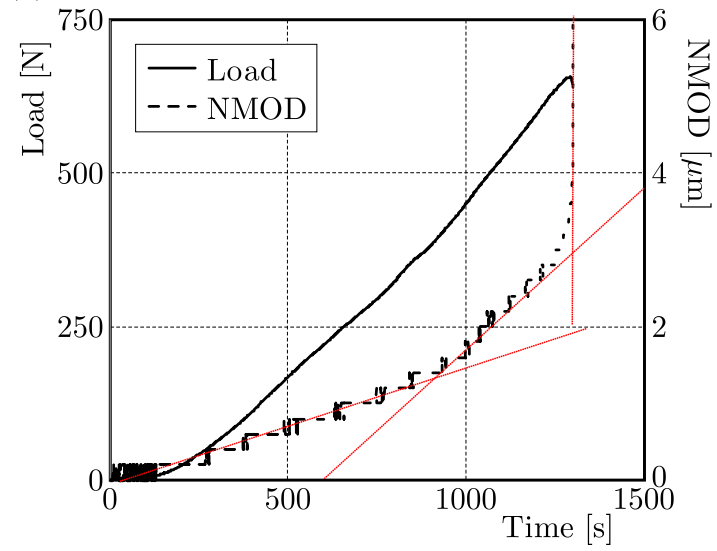

(b)

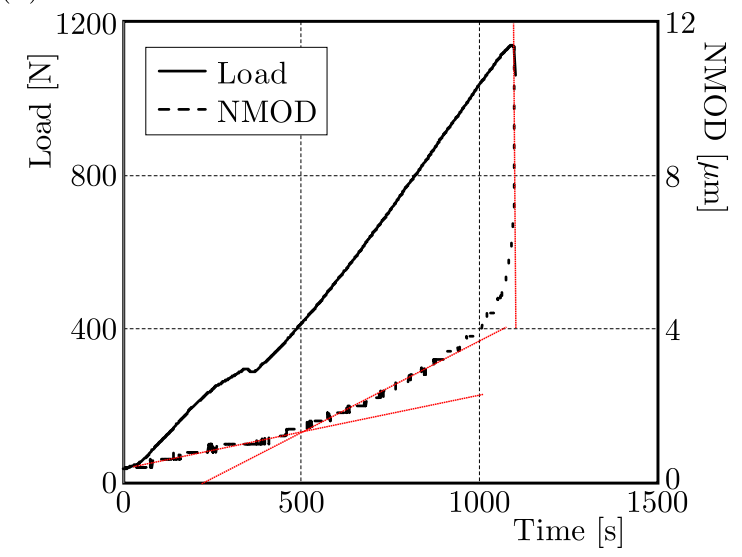

Fig. 12. The time evolution of the load induced in juxtaposition to the respective one of the NMOD, as recorded by the clip-gauge for a specimen with $b \times h=20 \times 20 \mathrm{~mm}^{2}$ (a) and $b \times h=25 \times 25 \mathrm{~mm}^{2}$ (b)

In an effort to detect a purely mechanical quantity that could, perhaps, offer such hints (i.e., warnings of upcoming catastrophic fracture), the time-variation of the NMOD, as obtained from the clip-gauge, is plotted in Fig. 12 in juxtaposition to the respective load-time variation (for the same as previously two specimens). In good qualitative accordance with the variation of the PSC-values, the NMOD-time curve consists, for both specimens, of three well distinguishable intervals: one of smooth and almost linear increase, a second one, which is again more or less linear but of definitely higher slope and, finally, a third one with very steep slope (as the load induced approaches its ultimate value). 


\subsection{Focusing attention on the very last loading stages}

The analysis of the PSC and NMOD data recorded during all tests of the present experimental protocol indicated clearly that the most dramatic changes of both quantities take place during the very last loading stage (and the same is true for the time variation of the acoustic activity, as it will become evident in next Sections). The duration of this stage does not exceed $10 \%$ of the tests duration. Given that the changes within this interval are very abrupt, it is quite possible that critical details of damage evolution could be shadowed by the extremely "condensed" nature of the events. It was thus decided to focus attention on this ultimate time-interval by taking advantage of an alternative mode for representing the respective experimental data. In this direction, the time evolution of PSC, NMOD and also of the acoustic activity are considered versus the $\left(t_{f}-t\right)$ parameter in semi-logarithmic scale, where $t_{f}$ is the time instant at which the specimen failed by catastrophic crack propagation.

Following the above concept, the time variation of the load and the PSC (both normalized over their respective maximum values) are plotted in Fig. 13 versus the $\left(t_{f}-t\right)$ parameter in juxtaposition to the acoustic activity as it is represented by the time rate of the number of acoustic hits detected by the acoustic sensor located just above the notch. The plots are drawn in a semi-logarithmic scale for the two specimens discussed in the previous Sections.
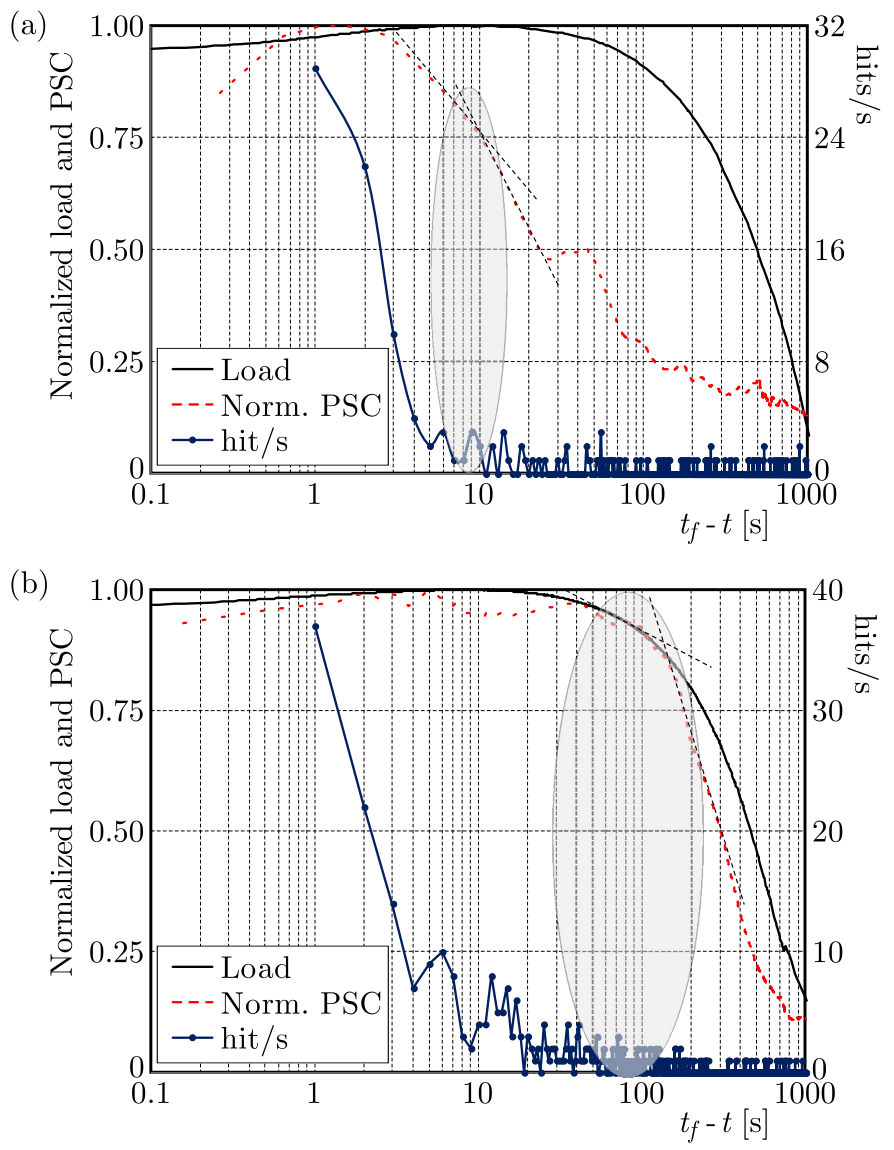

Fig. 13. The time evolution of the load induced (normalized over its maximum value) in juxtaposition to the respective ones of the PSC recorded (also normalized over its maximum value) and of the number of hits recorded per second for a specimen with $b \times h=20 \times 20 \mathrm{~mm}^{2}$ (a) and $b \times h=25 \times 25 \mathrm{~mm}^{2}$ (b). The quantities are plotted in semi-logarithmic scale against the $\left(t_{f}-t\right)$ parameter to better enlighten the phenomena at the very last loading levels

Figure 13a corresponds to the specimen with $b \times h=20 \times 20 \mathrm{~mm}^{2}$. Concerning the acoustic activity, it can be seen that the specimen is almost "silent" for $t_{f}-t>10 \mathrm{~s}$. The number of 
hits recorded per second is almost equal to zero. Then, at the time instant $t_{f}-t=10 \mathrm{~s}$, the rate of acoustic hits recorded by the acoustic sensor starts increasing dramatically. It is very interesting to observe that the specific time instant corresponds to a clear decrease in the slope of the respective time-PSC curve (see the shadowed ellipse in Fig. 13a).

The overall time evolution of the above quantities (load, PSC and rate of acoustic hits) is qualitatively similar also for the specimens of the second class (i.e., the ones with $b \times h=$ $25 \times 25 \mathrm{~mm}^{2}$ ), as it is shown in Fig. 13b. However, the precursor phenomena designated by the changes of the PSC and the rate of acoustic hits appear now somehow earlier, i.e., at about $t_{f}-t=100 \mathrm{~s}$. Indeed, the specimen is now "silent" for the time interval $t_{f}-t>100 \mathrm{~s}$. Then, the acoustic activity starts increasing relatively smoothly, and only at $t_{f}-t=10 \mathrm{~s}$ it starts increasing dramatically. Again, the time instant at which the acoustic activity is amplified for the first time (i.e., the instant $t_{f}-t=100 \mathrm{~s}$ ) is very close to the time instant at which the respective time-PSC curve changes its slope (see the shadowed ellipse in Fig. 13b). Indeed, while for $t_{f}-t>100 \mathrm{~s}$ the PSC increases monotonically (following the respective behaviour of the load), at $t_{f}-t=100 \mathrm{~s}$ the rate of the increase of the PSC changes and its value tends to be stabilized. For this specific specimen, at the onset of "explosive" amplification of the acoustic activity (at $t_{f}-t=10 \mathrm{~s}$ ) the value of the PSC has been already stabilized and it starts decreasing, indicating, obviously, generation of cracks of a higher order (due to the coalescence of already existing cracks of smaller length) which interrupt the electric paths.

Along the same lines, the time variation of the acoustic activity is plotted in Fig. 14 in juxtaposition to the respective variation of the time rate of the NMOD recorded with the aid of clip-gauge. The plots are again considered versus the $\left(t_{f}-t\right)$ parameter in semi-logarithmic scale for the same specimens as previously. The identity of the curves corresponding to the acoustic activity and to the rate of change of the NMOD-values is quite striking.
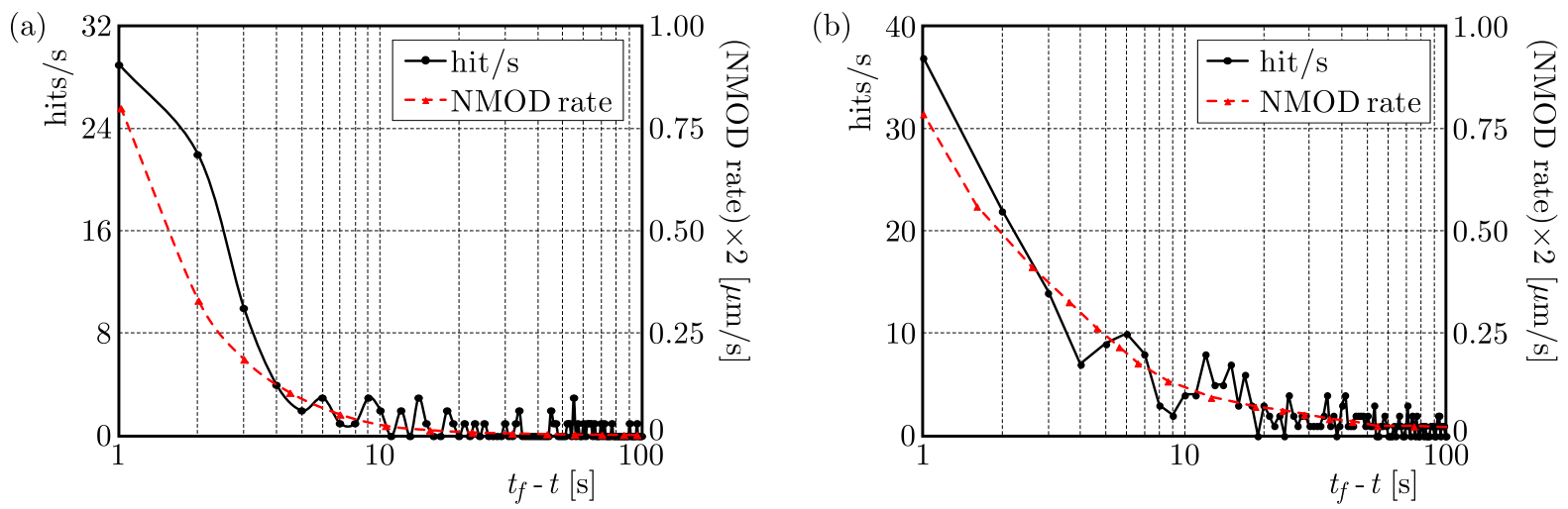

Fig. 14. The time evolution of the NMOD rate in juxtaposition to the respective one of the number of hits recorded per second for a specimen with $b \times h=20 \times 20 \mathrm{~mm}^{2}$ (a) and $b \times h=25 \times 25 \mathrm{~mm}^{2}$ (b). The quantities are plotted in semi-logarithmic scale against the $\left(t_{f}-t\right)$ parameter to better enlighten the phenomena at the very last loading levels

Concerning now the $I b$-value, its time variation is plotted against the $\left(t_{f}-t\right)$ parameter (again in semi-logarithmic scale), in Fig. 15a, for a characteristic specimen, belonging to the first class. It is seen that the $I b$-value starts decreasing towards a level equal to one (considered as an indication that the system enters its "critical stage") at about the same time instant of the slope change of the respective $\left(t_{f}-t\right)$-PSC plot. The respective graph for a specimen of the second class, shown in Fig. 15b, is qualitatively similar. The main difference is that now the decrease of the $I b$-value towards levels equal to one starts somehow earlier, i.e., at $\left(t_{f}-t\right)$ values around $100 \mathrm{~s}$ (again in accordance with the slope change of the respective $\left(t_{f}-t\right)$-PSC plot). 
(a)

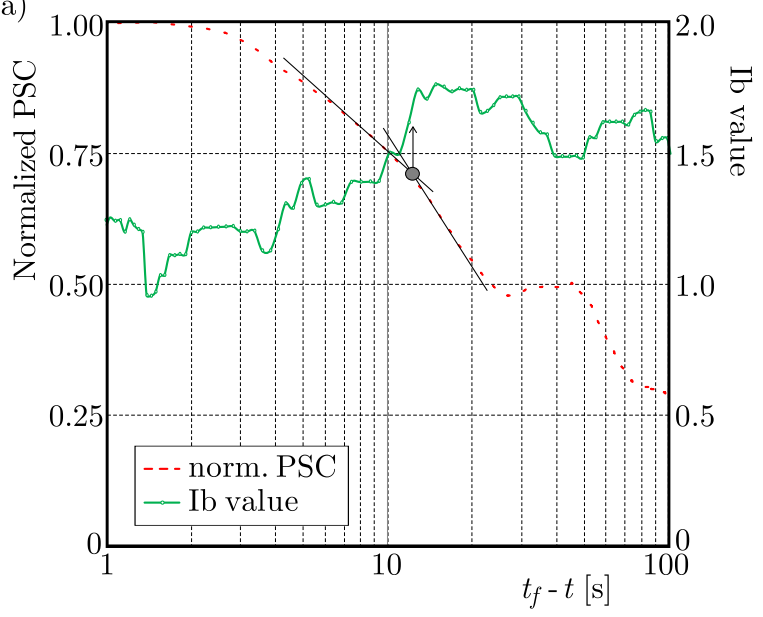

(b)

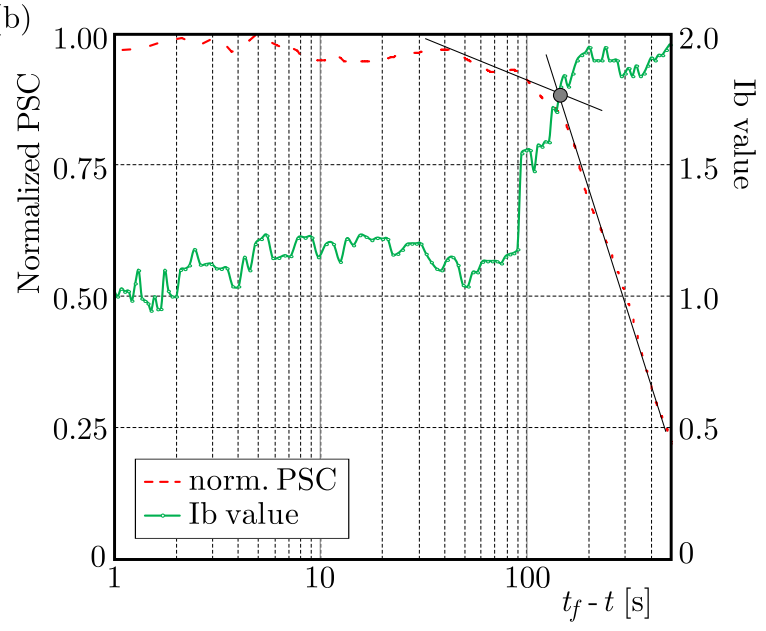

Fig. 15. The time variation of the $I b$-value in juxtaposition to the respective one of the PSC for a specimen with $b \times h=20 \times 20 \mathrm{~mm}^{2}$ (a) and $b \times h=25 \times 25 \mathrm{~mm}^{2}$ (b)

\subsection{Classification of the cracking modes}

Taking advantage of the characteristics of the acoustic activity, an attempt is now made to classify the cracks generated during the whole loading procedure, focusing again attention on the very last loading stages. Following Ohno and Ohtsu (2010) and Aggelis (2011), the Average Frequency of the acoustic hits is plotted in Fig. 16 either against the RA (Rise Time per Amplitude) parameter (left column of figures) or against the Rise Time (right column of figures) for the above two specimens. It is clear from Fig. 16a (corresponding to the specimen of the first class) that during $t_{f}-t>10 \mathrm{~s}$ time interval the acoustic activity is definitely due to exclusively tensile micro-cracking (recall that $t_{f}-t=10 \mathrm{~s}$ corresponds to the time instant at which the dramatic changes of the electric and acoustic activity appear for the specific specimen). Indeed, in this time interval the values of both the RA-parameter and the respective Rise Time are very small (tending to zero). It is only during the last ten seconds that events with increasing RA and Rise Time values appear while, at the same time, their average frequency decreases significantly, indicating that Mode II cracking or shear phenomena appear. This phenomenon is even clearer during the very last second of the loading procedure $\left(t_{f}-t<1 \mathrm{~s}\right)$.

The existence of a significant number of non-tensile cracks is, perhaps, somehow astonishing, especially for 3PB tests with pre-notched specimens for which fracture is attributed to tensile stresses around the crown of the notch. A possible explanation could attribute the existence of Mode II and mixed mode cracking (as well as of other type of shear phenomena) to the layered structure of the specific marble type. Indeed, the material layers are somehow wavy and, therefore, they are not uniformly oriented with respect to the loading axis resulting to irregular crack paths (mainly at the microscopic but also at the macroscopic level). These paths, at a given time instant, may be parallel to the material layers (within them), and a few moments later they may become normal to them. As a result, a variety of cracking modes appears (either tensile or shear or mixed mode), in spite of the fact that the macroscopic overall stress field consists of normal stresses (either tensile ones prevailing at the lower portion of the specimens, or compressive ones prevailing at the upper portion). The above described sequence of activation of damage mechanisms is in general agreement with what is widely accepted concerning the fracture of structural materials and the sequence of cracking modes (Aggelis et al., 2013), i.e., that "tensile stresses induce initial micro-cracking and later shear phenomena dominate as damage is being accumulated". 
(a)
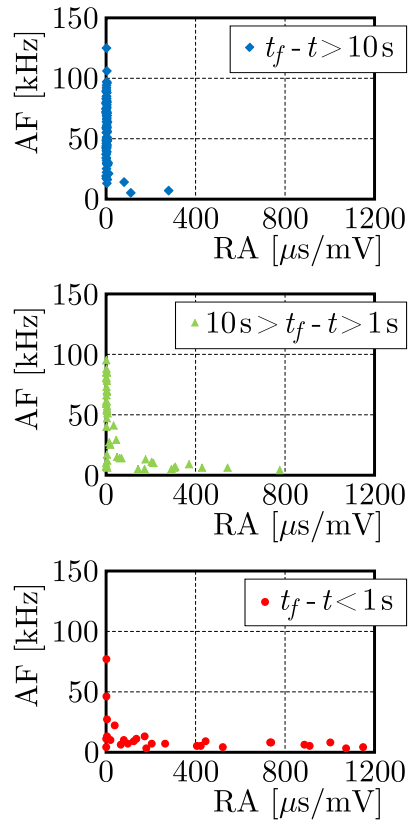

(b)
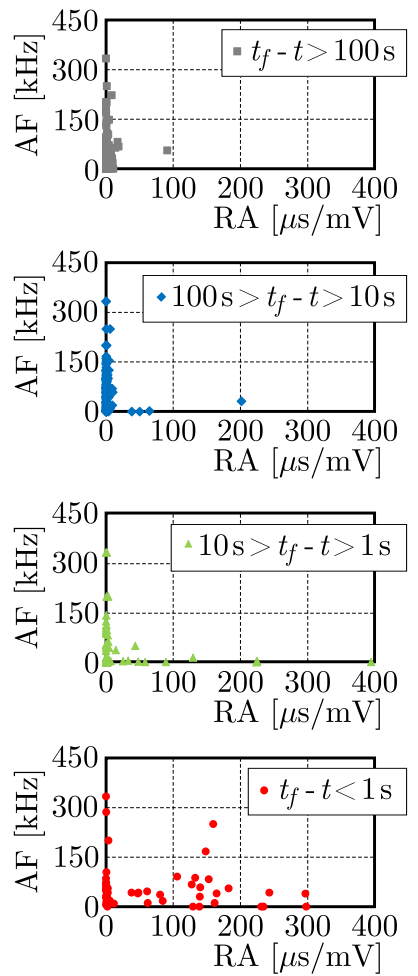
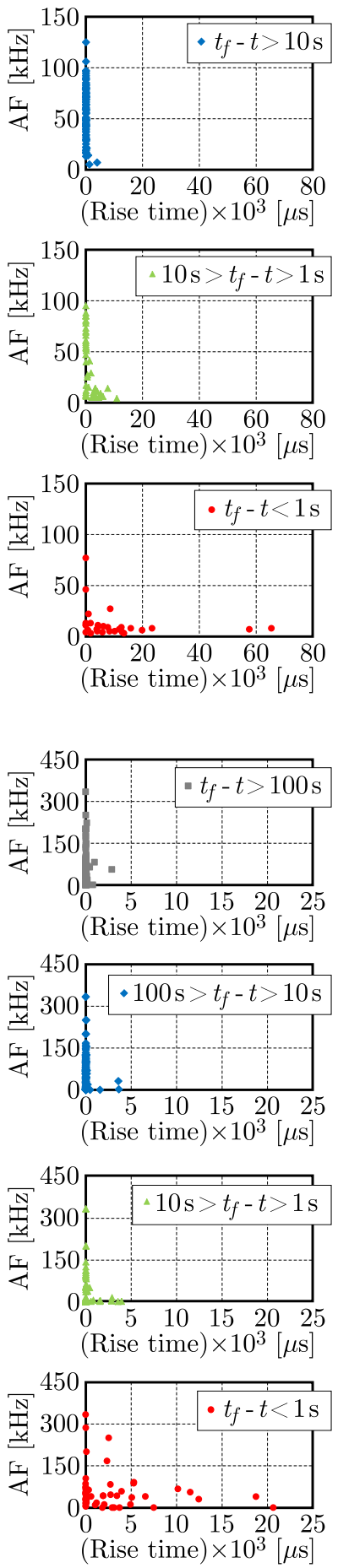

Fig. 16. The Average Frequency of the AE signals recorded versus their RA (Rise Time per Amplitude) parameter (left column) and versus their Rise Time (right column) for a specimen with $b \times h=20 \times 20 \mathrm{~mm}^{2}(\mathrm{a})$ and $b \times h=25 \times 25 \mathrm{~mm}^{2}(\mathrm{~b})$

The conclusions drawn for the specimen of the second class are almost identical, as it can be seen in Fig. 16b. The only difference is that now the generation of Mode II cracking or shear starts earlier, i.e., at about $t_{f}-t=100 \mathrm{~s}$ (again in full accordance with what was observed for the changes of the electric and acoustic activity and the time evolution of the NMOD rate). Exclusively for clarity reasons the duration of the specific test was split into four intervals. 


\section{Concluding remarks}

The response of notched marble beams to mechanical loading under $3 \mathrm{~PB}$ conditions was studied experimentally, using both traditional and innovative sensing techniques, in an effort to enlighten some critical aspects of their mechanical response and also to check whether it is possible to detect precursor phenomena that could act as signalling effects designating upcoming catastrophic failure.

Concerning the mechanical response, it was concluded that the NMOD-stress relation deviates from linearity from relatively early load levels, contrary to the respective load-deflection relation which is almost linear for the whole loading procedure. The above difference indicates the crucial role of the process zone developed around the crown of the notch. In spite of its very small relative size, this zone affects significantly the evolution of NMOD since the latter depends on the local stress field around the crown of the notch. On the other hand, due to its small size, this zone cannot influence the overall stress field which remains almost linear, explaining the linearity between load and deflection.

Along the same lines, it was pointed out that the slope of the stress-NMOD curve at its linear portion is significantly different from the respective slope predicted using the classical Fracture Mechanics tools. Although some differences were expected due to the fact that in the present protocol notched rather than cracked beams were tested, the magnitude of this difference (exceeding 100\%) was astonishing. Following Vardoulakis et al. (1998), this huge difference is attributed to the existence of an internal structure, which does not permit characterization of Dionysos marble as an amorphous material. As a result, such materials should be described using tools of gradient elasticity rather than traditional tools of Continuum Mechanics and Fracture Mechanics.

Another interesting finding of the present protocol is that the fracture stress determined by the 3PB tests (with either intact or pre-notched specimens) significantly overestimates the tensile fracture stress obtained by direct tension tests. This observation should be carefully taken into account by engineers since it is a common practice to determine tensile properties of very brittle geomaterials (like rocks and rock-like materials) using bending tests, since their laboratory implementation is quite simple.

Interesting conclusions were also drawn regarding the deformed shape of the specimens just before fracture. Indeed, it was quantitatively pointed out that the deformed shapes of the upper and lower edges of the beams were different. Although such a result should be expected due to the influence of the point load, it was the first time that this difference was experimentally quantified for a material like Dionysos marble, which is extensively used for construction of structural elements subjected to bending schemes.

Regarding the notch itself, it was concluded that its flanks remain linear with excellent accuracy. This observation permits a linear extrapolation of the data recorded at the mouth of the notch (i.e., the NMOD-values recorded by clip-gauges attached at the mouth of the notch) to its tip. As a result, techniques like that proposed by Knauf and Riedel (1981) for the determination of COD and its critical value for completely different materials can be used, also, for the determination of NOD and its critical value for brittle geo-materials like Dionysos marble.

Recapitulating, it became clear that although Dionysos marble is a very brittle material and its overall mechanical response in terms of classical strength of materials is more or less linear, its mechanical behaviour in the presence of notches cannot be adequately described in terms of linear elastic Continuum Fracture Mechanics.

Concerning now the second target of the present experimental protocol, i.e., the detection of precursor phenomena designating impending fracture, interesting conclusions were drawn for all three quantities considered, namely mechanical, electric and acoustic ones. To recapitulate 
these conclusions, all three quantities considered, i.e., the PSC, the rate of cumulative acoustic hits per second $(d N / d t)$ and the rate of NMOD, are plotted in Fig. 17 versus the "nominal" stress for a typical specimen of the class with $b \times h=20 \times 20 \mathrm{~mm}^{2}$. The similarity of all curves is striking: In spite of their completely different nature (electrical, acoustic and mechanical), all three quantities behave almost according to the same manner with respect to the stress level (or equivalently to the load induced). Initially (i.e., for low stress compared to the fracture one), they are either almost constant (PSC) or they increase very smoothly and, in any case, linearly $(d N / d t$ and NMOD). Then, at a stress level equal to about two thirds of its ultimate value, a dramatic change is observed and the respective curves start increasing according to a completely different slope, tending to become almost vertical.

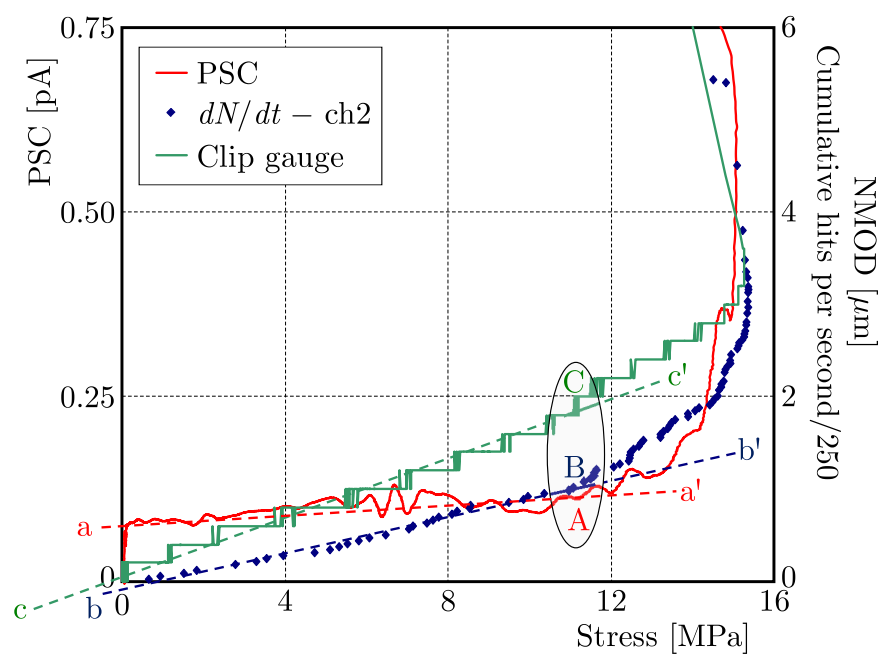

Fig. 17. The evolution of the electric and acoustic activity versus the axial "nominal" stress in juxtaposition to the respective one of the NMOD for a typical specimen with $b \times h=20 \times 20 \mathrm{~mm}^{2}$, highlighting the existence of mutually compatible precursor indicators

Considering the above experimental evidence, it is obvious that a relation exists between the mechanical response of Dionysos marble and the respective electric and acoustic processes taking place within the specimen volume. This is schematically shown in Fig. 17 where the narrow shaded elliptic area encompasses all three phenomena:

(i) Deviation of the stress-PSC plot from its almost constant portion, represented by line $a a^{\prime}$ (point $A$ ).

(ii) Deviation of the stress-dN/dt plot from its linear portion, represented by line $b b^{\prime}$ (point $B$ ).

(iii) Deviation of the stress-NMOD rate plot from its linear portion, represented by line $c c^{\prime}$ (point $C$ ).

Taking now into account that the increase of the PSC definitely designates the onset of intense micro-cracking (Triantis et al., 2006; Anastasiadis et al., 2007) and also that the quantity $d N / d t$ is directly related to the number of micro-cracks generated within the specimen's volume, it can be safely concluded that: At least for the tests of the specific experimental protocol, irreversible processes start taking place at load levels equal to about two thirds of the fracture stress, at rates definitely different from those of the earlier loading stages.

The second very interesting conclusion (which was drawn by considering the very last loading stages through the alternative (inversed) representation of the "time arrow", see Figs. 13-15) is that a little before the final fracture, the time-PSC plot exhibits well distinguishable changes (in good accordance with the remaining quantities considered), that could be related to the generation of fatal cracks all along the active cross section of the specimen, indicating the exhaust of its load-carrying capacity. From this point on, and in spite of the fact that the load 
level will increase slightly (perhaps due to inertia effects), the specimen should be considered fractured. Therefore, these changes of the PSC readings could be used for the determination of the critical NMOD, curing the problems mentioned previously in Section 3, regarding the actual point of the NMOD-stress curve that corresponds to the critical NMOD value.

Along the same lines, it is very interesting to note that the above mentioned changes of the time-PSC plot, designating the exhaust of the specimen's load-carrying capacity, are in good agreement with the respective hints provided by the time evolution of the $I b$-value.

Concluding, it can be said that taking advantage of innovative experimental techniques, interesting data can be pumped from the interior of loaded elements, uniquely related to failure mechanisms activated well before any macroscopic fracture is observed.

Research in progress, with double edge notched (DENT) dog-bone marble specimens under direct tension, definitely supports the conclusions of the present study (Kourkoulis et al., 2018). What is more important is that the potentialities of the PSC technique are also supported by a protocol in progress with much more complex structures, as it is for example marble epistyles mutually interconnected with the aid of titanium elements and suitable cementitious materials subjected to pure shear. Although this project is still in progress (given that quite a few difficulties generated by the existence of interfaces are to be overcome), the results are quite encouraging (Pasiou et al., 2018).

Taking into account the conclusions just mentioned, it is indicated that it is worth exploring further the potentialities of the PSC technique to be used as an alternative Structural Health Monitoring tool. This statement is further supported by taking into account the very low cost of the sensors required to record the electric current and also their relatively small size. Especially for restoration projects of ancient monuments, these characteristics of the PSC technique are very attractive because usually there are too many elements restored (thus many sensors are required) and also the aesthetic aspect (and, therefore, the small size of the sensors) is of utmost importance. The excellent agreement of the output of the PSC technique with the respective ones of the AE technique provides a very reliable calibration tool for the PSC technique, considering that nowadays Acoustic Emission is a mature, widely used sensing technique based on well founded natural basis.

Clearly, before definite conclusions are drawn, the qualitative correlations enlightened in the present protocol must be further studied for a much wider class of materials and tests (tension, compression and shear) in the direction of providing quantitative rather than qualitative correlations that could permit calibration of the outcomes of the PSC technique against those of the Acoustic Emissions technique.

\section{Acknowledgements}

This research was co-financed by the EU (European Social Fund-ESF) and Greek national funds through the Operational Program "Education and Lifelong Learning" of the National Strategic Reference Framework (NSRF) - Research Funding Program: THALES: Reinforcement of the interdisciplinary and/or inter-institutional research and innovation. The authors gratefully acknowledge the support.

The authors express their sincere thanks to the two anonymous reviewers of the present manuscript. Their comments and suggestions were extremely valuable in the direction of significantly improving the quality and scientific integrity of the present manuscript.

\section{References}

1. AgGelis D.G., 2011, Classification of cracking mode in concrete by acoustic emission parameters, Mechanics Research Communications, 38, 153-157 
2. Aggelis D.G., Mpalaskas A., Matikas T.E., 2013, Acoustic signature of different fracture modes in marble and cementitious materials under flexural load, Mechanics Research Communications, 47, 39-43

3. Anastasiadis C., Stavrakas I., Triantis D., Vallianatos F., 2007, Correlation of pressure stimulated currents in rocks with the damage variable, Annals of Geophysics, 50, 1-6

4. Andrianopoulos N.P., Kourkoulis S.K., Saragas S., 1997, COD measurements and optimum exploitation of metal matrix composites for aerospace applications, Engineering Fracture Mechanics, 57, 565-576

5. BAŽAnt Z.P., 1984, Size effect in blunt fracture: concrete, rock, metal, ASCE Journal of Engineering Mechanics, 110, 518-535

6. Blanchette Y., Dickson J.I., Bassim M.N., 1984, The use of acoustic emission to evaluate critical values of $K$ and $J$ in 7075-T651 aluminum alloy, Engineering Fracture Mechanics, 20, 359-371

7. Brynk T., Laptiev A., Tolochyn O., Pakiela Z., 2012, The method of fracture toughness measurement of brittle materials by means of high speed camera and DIC, Computational Materials Science, 64, 221-224

8. Carpinteri A., 1989, Decrease of apparent tensile and bending strength with specimen size: Two different explanations based on fracture mechanics, International Journal of Solids and Structures, 25, 407-429

9. Erdogan F., Sin G.C., 1963, On the crack extension in plates under plane loading and transverse shear, ASME Journal of Basic Engineering, 85D, 519-527

10. Exadaktylos G.E., Vardoulakis I., Kourkoulis S.K., 2001, Influence of nonlinearity and double elasticity on flexure of rock beams - I. Technical theory, International Journal of Solids and Structures, 38, 4091-4117

11. Hashida T., 1993, Fracture toughness testing of core-based specimens by acoustic emission, International Journal of Rock Mechanics and Mining Sciences and Geomechanics Abstracts, 30, 61-69

12. Kishinoue T., 1990, An experiment on the progression of fracture (preliminary rep.), Journal of Acoustic Emission, 9, 177-180

13. Knauf C., Riedel H., 1981, A comparative study on different methods to measure the crack opening displacement, Proceedings of the 5th International Conference on Fracture (ICF5), Cannes, France, 2547-2553

14. Korres M., Bouras H., 1983, Study for the Restoration of the Parthenon, Ministry of Culture and Science, Committee for the Preservation of the Acropolis Monuments, Athens, Greece

15. Kourkoulis S.K., Exadaktylos G. E., Vardoulakis I., 1999, U-notched Dionysos-Pentelicon marble beams in three point bending: The effect of nonlinearity, anisotropy and microstructure, International Journal of Fracture, 98, 369-392

16. Kourkoulis S.K., Ganniari-Papageorgiou E., 2010, Experimental study of the size-and shape-effects of natural building stones, Construction and Building Materials, 24, 803-810

17. Kourkoulis S.K., Pasiou E.D., Dakanali I., Stavrakas I., Triantis D., 2018, Notched marble plates under tension: Detecting pre-failure indicators and predicting entrance to the "critical stage", Fatigue and Fracture of Engineering Materials and Structures, 41, 776-786

18. Kyriazopoulos A., Anastasiadis C., Triantis D., Brown J.C., 2011, Non-destructive evaluation of cement-based materials from pressure-stimulated electrical emission - Preliminary results, Construction and Building Materials, 25, 1980-1990

19. Labuz J.F., Cattaneo S., Chen L.H., 2001, Acoustic emission at failure in quasi-brittle materials, Construction and Building Materials, 15, 225-233

20. Maji A., Shah S.P., 1988, Process zone and acoustic-emission measurements in concrete, Experimental Mechanics, 28, 27-33 
21. Markides Ch.F., Kourkoulis S.K., 2016, 'Mathematical' cracks versus artificial slits: Implications in the determination of fracture toughness, Rock Mechanics and Rock Engineering, 49, $707-729$

22. Muralidhara S., Prasad B.R., Eskandari H., Karihaloo B.L., 2010, Fracture process zone size and true fracture energy of concrete using acoustic emission, Construction and Building Materials, 24, 479-486

23. OBert L., 1941, Use of subaudible noise for the prediction of rock bursts, Report R13555, U.S. Bureau of Mines, Washington D.C.

24. Oнno K., Oнtsu M., 2010, Crack classification in concrete based on acoustic emission, Construction Building Materials, 24, 2339-2346

25. Ohtsu M., 2010, RILEM TC 212-ACD: Acoustic emission and related NDE techniques for crack detection and damage evaluation in concrete, Materials and Structures, 43, 1187-1189

26. Pasiou E.D., Stavrakas I., Triantis D., Kourkoulis S.K., 2018, Marble epistyles under shear: An experimental study of the role of "relieving space", submitted to Frontiers of Structural and Civil Engineering

27. Rao M.V.M.S., Prasanna Lakshmi K.J., 2005, Analysis of b-value and improved b-value of acoustic emissions accompanying rock fracture, Current Science, 89, 1577-1582

28. Sachse W., Yamaguchi K., Roget J., 1991, Acoustic Emission: Current Practice and Future Directions, STP1077, ASTM

29. Saragas S., Kourkoulis S.K., Andrianopoulos N.P., 1996, A comparative study of COD measurements for novel metal matrix composites and alloys, International Journal of Fracture, 79, 179-187

30. Shiotani T., Fuji K., Aoki T., Amou K., 1994, Evaluation of progressive failure using AE sources and improved b-value on slope model tests, Progress in Acoustic Emission VII, 7, 529-534

31. Shiotani T., Yuyama S., Li Z.W., Ohtsu M., 2001, Application of the AE improved b-value to qualitative evaluation of fracture process in concrete-materials, Journal of Acoustic Emission, 19, 118-133

32. Siн G.C., 1973, Some basic problems in Fracture Mechanics and new concepts, Engineering Fracture Mechanics, 5, 365-377

33. Slifkin L., 1993, Seismic electric signals from displacement of charged dislocations, Tectonophysics, 224, 149-152

34. Stavrakas I., Anastasiadis C., Triantis D., Vallianatos F., 2003, Piezo stimulated currents in marble samples: Precursory and concurrent-with-failure signals, Natural Hazards and Earth System Sciences, 3, 243-247

35. Stergiopoulos C., Stavrakas I., Triantis D., Vallianatos F., Stonham J., 2015, Predicting fracture of mortar beams under three-point bending using non-extensive statistical modelling of electric emissions, Physica A, 419, 603-611

36. Sulem J., Vardoulakis I., 1990, Bifurcation analysis of the triaxial test on rock specimens. A theoretical model for shape and size effect, Acta Mechanica, 83, 195-212

37. Sutton M.A., Cheng M., Peters W.H., Chao Y.J., McNeill S.R., 1986, Application of an optimized digital correlation method to planar deformation analysis, Image and Video Computing, 4, 143-150

38. Sutton M.A., McNeill S.R., Helm J.D., Chao Y.J., 2000, Advances in two-dimensional and three-dimensional computer visio, [In:] Photomechanics, Rastogi P.K. (Edit.), Topics in Applied Physics, Springer, Berlin, 77, 323-372

39. Tassogiannopoulos A.G., 1986, A contribution to the study of the properties of structural natural stones of Greece (in Greek), Ph.D. Dissertation, Supervisor: A. Koroneos, National Technical University of Athens, Greece 
40. Tada H., Paris P.C., Irwin G.R., 1973, The Stress Analysis of Cracks Handbook, Del Research Corporation

41. Theocaris P.S., Kardomateas G., Andrianopoulos N.P., 1982, Experimental study of the T-criterion in ductile fractures, Engineering Fracture Mechanics, 17, 439-445

42. The Venice Charter: International charter for the conservation and restoration of monuments and sites, 1964, IInd International Congress of Architects and Technicians of Historic Monuments, ICOMOS, Venice, Italy

43. Triantis D., Anastasiadis C., Vallianatos F., Kyriazis P., Nover G., 2007, Electric signal emissions during repeated abrupt uniaxial compressional stress steps in amphibolite from KTB drilling, Natural Hazards and Earth System Science, 7, 149-154

44. Triantis D., Stavrakas I., Anastasiadis C., Kyriazopoulos A., Vallianatos F., 2006, An analysis of Pressure Stimulated Currents (PSC) in marble samples under mechanical stress, Physics and Chemistry of the Earth, 31, 234-239

45. Triantis D., Stavrakas I., Kyriazopoulos A., Hloupis G., Agioutantis Z., 2012, Pressure stimulated electrical emissions from cement mortar used as failure predictors, International Journal of Fracture, 175, 53-61

46. Vallianatos F., Tzanis A., 1999, On possible scaling laws between electric earthquake precursors (EEP) and earthquake magnitude, Geophysical Research Letters, 26, 2013-2016

47. Vardoulakis I., Exadaktylos G.E., Kourkoulis S.K., 1998, Bending of marble with intrinsic length scales: A gradient theory with surface energy and size effects, Journal de Physique IV, 8, 399-406

48. Vardoulakis I., Kourkoulis S.K., 1997, Mechanical properties of Dionysos marble, Final report of the Environment Project EV5V-CT93-0300: Monuments Under Seismic Action, National Technical University of Athens, Athens, Greece

49. Varotsos P.A., 2005, The Physics of Seismic Electric Signals, TerraPub, Tokyo, Japan

50. Whitworth R.W., 1975, Charged dislocations in ionic crystals, Advances in Physics, 24, 203-304

51. Wu C.H., 1978, Fracture under combined loads by the maximum-energy release rate criterion, Journal of Applied Mechanics, 45, 553-558

52. Yoffe E. H., 1951, The moving Griffith crack, Philosophical Magazine, 42, 739-750

53. Zambas K., 1994, Study for the Restoration of the Parthenon, Vol. 3b, Ministry of Culture, Committee for the Preservation of the Acropolis Monuments, Athens, Greece 